\title{
Asymptotic Matrix Theory of Bragg Fibers
}

\author{
Yong Xu, George X. Ouyang, Reginald K. Lee, Member, IEEE, and Amnon Yariv, Life Fellow, IEEE
}

\begin{abstract}
We developed a matrix theory that applies to any cylindrically symmetric fiber surrounded with Bragg cladding, which includes both the Bragg fibers and the recently proposed dielectric coaxial fibers. In this formalism, an arbitrary number of inner dielectric layers are treated exactly and the outside cladding structure is approximated in the asymptotic limit. An estimate of the radiation loss of such fibers is given. We compare the asymptotic results with those obtained from the finite difference time domain (FDTD) calculations and find excellent agreement between the two approaches.
\end{abstract}

Index Terms-Approximation methods, Bragg scattering, optical fiber dispersion, optical fiber losses, optical fibers, optical waveguide theory, periodic structures.

\section{INTRODUCTION}

I $\mathrm{N}$ conventional optical fibers, light confinement is achieved through total internal reflection and photons propagate mainly in the high index center core. A completely different confinement mechanism, Bragg reflection, provides an alternative way of guiding photons. This possibility was first pointed out by Yeh et al. in [1], where the concept of Bragg fiber was proposed. The experimental fabrication of Bragg fibers has been recently reported [2]. Fig. 1 is the schematic of a Bragg fiber, which consists of a center dielectric core surrounded by cladding layers with alternating high and low refractive indexes.

Because Bragg fibers and conventional optical fibers utilize different guiding mechanisms, it is not surprising that Bragg fibers offer many possibilities that are difficult to achieve in conventional fibers. One such example is the possibility of guiding light in air, which has attracted much recent interest [2]-[6]. In a Bragg fiber with large cladding index contrast, light can be confined mostly within the center air core, which can lead to lower propagation loss and reduce the threshold for nonlinear effects. A Bragg fiber can also be designed to support a single guided mode without azimuthal dependence. In contrast with the fundamental mode in conventional fibers, which is always doubly degenerate, these guided Bragg fiber modes are truly single mode. Consequently, many undesirable polarization dependent effects can be completely eliminated in Bragg fibers [6].

The analysis of Bragg fibers, however, is much more complicated than that of conventional fibers. In the matrix formalism of [1], Yeh et al. used four independent parameters to describe the solution of Maxwell equations in each layer of the Bragg fiber and the parameters in neighbor dielectric layers were related via a $4 \times 4$ matrix (see also Section II-A of this paper). Un-

\footnotetext{
Manuscript received June 26, 2001. This work was supported in part by the Office of Naval Research.

The authors are with the Department of Applied Physics, California Institute of Technology, Pasadena, CA 91125 USA (e-mail: yong@its.caltech.edu).

Publisher Item Identifier S 0733-8724(02)02199-0.
}

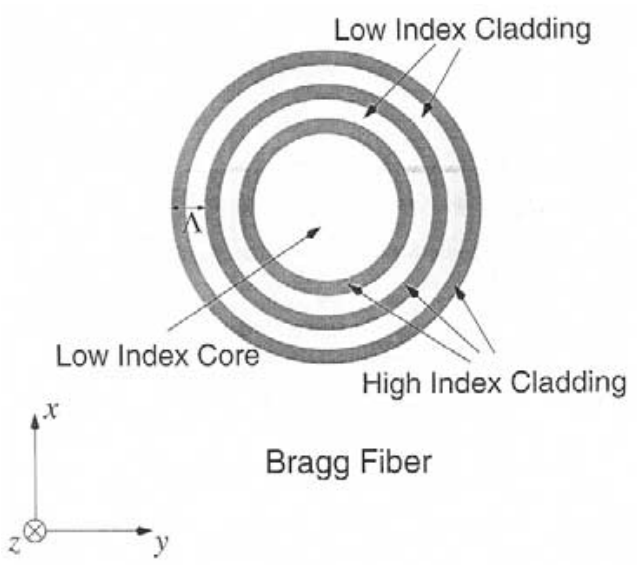

Fig. 1. Schematic of a Bragg fiber. The center of the Bragg fiber is composed of low-index material, including air. The Bragg fiber cladding consists of alternating layers of dielectric media with high- and low-refractive indexes.

like the case of conventional fibers, in this approach, the confined modes in a Bragg fiber were treated as quasi-modes whose propagation constant and field distribution were found by minimizing the radiation loss [1]. For transverse electric (TE) and transverse magnetic (TM) modes, the minimization procedure is relatively easy, because the radiation loss depends only on a single variable, the propagation constant $\beta$. For other types of guided modes, this approach becomes much more complicated. The extra complexity associated with this matrix approach is due to the difficulty in finding the eigenmode in fiber cladding layers. For a planar air core Bragg waveguide, the eigen solution that decays in the cladding structure can be easily found according to the Bloch theorem [8]. For a cylindrically symmetric Bragg fiber, which is, strictly speaking, not periodic and for which the Bloch theorem does not apply, we cannot single out an eigen solution that decays in the fiber cladding layers. As a result, it is no longer feasible to find an exact analytical equation that determines mode dispersion by matching the cladding solution and core solution at the waveguide core-cladding interface, as in the case of conventional fibers [7] or planar Bragg waveguides [8].

The problems associated with finding analytical solutions for Bragg fibers were partially solved by using the asymptotic analysis in [9]. The main observation is that in the asymptotic limit, the exact solutions of Maxwell equations, which take the form of Bessel functions, can be approximated as $\exp (i k r) / \sqrt{r}$ and $\exp (-i k r) / \sqrt{r}[10]$. In this form, the solutions in Bragg fiber cladding resemble those in planar Bragg stacks and eigen solutions in the fiber claddings can be similarly found. The solutions in the center core are still given by Bessel functions and the eigenmode equation is found by matching the cladding solutions with core solutions at the core-cladding interface. As the example in [9] demonstrated, the difference between the asymptotic results and results obtained from minimizing radiation loss 
is less than $2 \%$ for a Bragg fiber with a small air core radius comparable to the wavelength. The drawback is that this simple asymptotic analysis may fail if the Bragg fiber core becomes too small and it is difficult to estimate the accuracy of the asymptotic results.

In Section II-C, we extend the analysis in [9] into an asymptotic matrix formalism, in which the first several dielectric layers are treated exactly and the rest of the dielectric cladding structures is approximated in the asymptotic limit. This new approach has the obvious advantage of being more accurate, because the asymptotic approximation works better as the radius $r$ becomes larger. In fact, we can use this method to find the dispersion relation of Bragg fibers within any desired precision simply by increasing the number of inner layers that are treated exactly. Equally important, although the original asymptotic analysis may fail for Bragg fibers with air core too small, the current approach can always generate satisfactory results by treating several inner layers exactly. The accuracy of the asymptotic approximation can also be estimated by comparing results obtained from treating different number of inner layers exactly, as will be demonstrated in this paper.

As mentioned previously, an air core Bragg fiber may reduce the photon propagation loss below what can be achieved in conventional fibers. Basically, there are two sources that contribute to the propagation loss in Bragg fiber, the material absorption loss and the radiation loss. The material absorption loss depends on the choice of dielectric medium and is not considered in this paper. On the other hand, the radiation loss mainly depends on the index contrast between the cladding media and the number of cladding pairs. In principle, the radiation loss can be reduced below any given number simply by using a large enough number of cladding pairs. However, using too many cladding pairs is generally undesirable or even impractical. In Section III, we give an estimate about the number of cladding pairs necessary to achieve $0.2-\mathrm{dB} / \mathrm{km}$ radiation loss, as a comparison with typical conventional fiber propagation loss.

Aside from the aforementioned analytical approaches, the guided Bragg fiber modes can also be found by numerically solving Maxwell equations, using various algorithms such as finite difference time domain (FDTD) method [11] and plane wave expansion method [12]. In Section IV, we use a two-dimensional (2-D) FDTD algorithm [13] to find the modal dispersion of an air core Bragg fiber and compare the results with those obtained from the asymptotic matrix formalism. In the same section, we also use the asymptotic approach to calculate the field distribution of guided modes.

It is also worth mentioning that the asymptotic matrix algorithm in Section II can also be directly applied to other cylindrically symmetric structures surrounded by Bragg cladding layers. One such example is the recently proposed dielectric coaxial fibers [6], which are formed by inserting a high refractive index rod into the air core of Bragg fibers. The asymptotic studies of coaxial fibers shall be the subject of another paper.

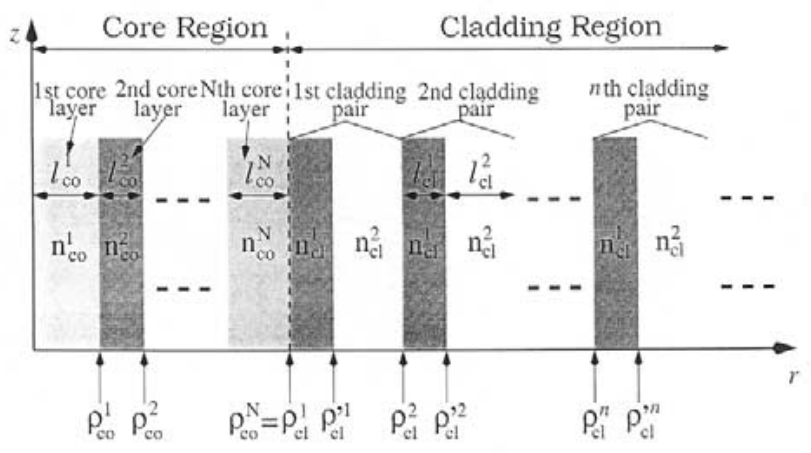

Fig. 2. Schematic of the $r-z$ cross section of a Bragg fiber. The dielectric layers of the Bragg fiber are classified into two regions, the core region and the cladding region, which are separated by the dash line in the figure. The core region consist of $N$ concentric layers each with refractive index $n_{c o}^{i}$ and thickness $l_{c o}^{i}, i=1 \ldots N$. The fiber cladding region is composed of pairs of alternating layers of different dielectric materials. Layer type I has refractive index $n_{\mathrm{cl}}^{1}$ and thickness $l_{\mathrm{cl}}^{1}$. Layer type II has refractive index $n_{\mathrm{cl}}^{2}$ and thickness $l_{\mathrm{cl}}^{2}$.

\section{ASYMPTOTIC MATRIX FORMALISM}

\section{A. Solution in the Core Region}

We begin our analysis by separating a Bragg fiber into two parts, with a core region and a cladding region as shown in Fig. 2. The fiber core region consists of the first $N$ concentric dielectric layers, which includes the center low index core. In this asymptotic matrix formalism, the exact solutions of Maxwell equations describe the fields in the core region. It should be emphasized that the refractive index and thickness of layers in the core region can be chosen arbitrarily. For the $i$ th layer, we respectively use $n_{\mathrm{CO}}^{i}$ and $l_{\mathrm{co}}^{i}$ to denote its refractive index and layer thickness, as shown in Fig. 2.

If we take the $z$ axis (see Fig. 2) as the direction of propagation, due to the translational symmetry, every field component has the following form [1]:

$$
\psi(r, \theta, z, t)=\psi(r, \theta) e^{i(\beta z-\omega t)}
$$

where $\psi$ can be $E_{z}, E_{r}, E_{\theta}, H_{z}, H_{r}$, and $H_{\theta}, \omega$ is the mode frequency, and $\beta$ is the propagation constant.

As in conventional fibers, the transverse field components can be represented by $E_{z}$ and $H_{z}$ [1], [7]

$$
\begin{aligned}
& E_{r}=\frac{i \beta}{\left(\frac{\omega^{2}}{c^{2}}\right) n^{2}-\beta^{2}}\left(\frac{\partial}{\partial r} E_{z}+\frac{\omega \mu_{0}}{\beta} \frac{\partial}{r \partial \theta} H_{z}\right) \\
& E_{\theta}=\frac{i \beta}{\left(\frac{\omega^{2}}{c^{2}}\right) n^{2}-\beta^{2}}\left(-\frac{\omega \mu_{0}}{\beta} \frac{\partial}{\partial r} H_{z}+\frac{\partial}{r \partial \theta} E_{z}\right) \\
& H_{r}=\frac{i \beta}{\left(\frac{\omega^{2}}{c^{2}}\right) n^{2}-\beta^{2}}\left(\frac{\partial}{\partial r} H_{z}-\frac{\omega \epsilon_{0} n^{2}}{\beta} \frac{\partial}{r \partial \theta} E_{z}\right) \\
& H_{\theta}=\frac{i \beta}{\left(\frac{\omega^{2}}{c^{2}}\right) n^{2}-\beta^{2}}\left(\frac{\omega \epsilon_{0} n^{2}}{\beta} \frac{\partial}{\partial r} E_{z}+\frac{\partial}{r \partial \theta} H_{z}\right)
\end{aligned}
$$

where $n$ is the refractive index of the dielectric medium.

Due to the cylindrical symmetry of Bragg fibers, we can take the azimuthal dependence of the field components as $\cos (l \theta)$ or $\sin (l \theta)$. For each $l$, the general solutions of $E_{z}$ and $H_{z}$ are the superposition of either $J_{l}(x)$ and $Y_{l}(x)$ or $I_{l}(x)$ and $K_{l}(x)$ [10]. In this section, we assume the solutions are given by $J_{l}(x)$ and 
$Y_{l}(x)$. As a result, the electromagnetic field at radius $r$, which is within the $i$ th core layer, can be written in the following matrix form [1]:

$$
\left[\begin{array}{c}
E_{z} \\
\frac{1}{i \beta} H_{\theta} \\
H_{z} \\
-\frac{1}{i \beta} E_{\theta}
\end{array}\right]=M\left(n_{\mathrm{co}}^{i}, k_{\mathrm{co}}^{i}, r\right)\left[\begin{array}{c}
A_{i} \\
B_{i} \\
C_{i} \\
D_{i}
\end{array}\right]
$$

The matrix $M\left(n_{\mathrm{co}}^{i}, k_{\mathrm{co}}^{i}, r\right)$ is defined as shown in (7) at the bottom of the page, where $k_{\mathrm{co}}^{i}=\sqrt{\left(n_{\mathrm{co}}^{i} \omega / c\right)^{2}-\beta^{2}}$. The coefficients $A_{i}, B_{i}, C_{i}$, and $D_{i}$ are constant within the $i$ th layer. We emphasize that as long as $\beta \leq n_{\text {co }}^{i} \omega / c$, (6) and (7) are the exact solution of Maxwell equations, with no approximation involved [1].

Once the electromagnetic field in the ith layer is known, we can easily find the field in the $(i+1)$ th layer by applying the condition that $E_{z}, E_{\theta}, H_{z}$, and $H_{\theta}$ are continuous at $r=\rho_{\mathrm{CO}}^{i}$, the interface between the $i$ th and $(i+1)$ th layer

$$
\left[\begin{array}{l}
A_{i+1} \\
B_{i+1} \\
C_{i+1} \\
D_{i+1}
\end{array}\right]=\mathbf{T}_{\mathbf{i}}\left[\begin{array}{l}
A_{i} \\
B_{i} \\
C_{i} \\
D_{i}
\end{array}\right]
$$

where the transfer matrix $\mathbf{T}_{\mathbf{i}}$ is

$$
\mathbf{T}_{\mathbf{i}}=\left[M\left(n_{\mathrm{co}}^{i+1}, k_{\mathrm{co}}^{i+1}, \rho_{\mathrm{co}}^{i}\right)\right]^{-1} M\left(n_{\mathrm{co}}^{i}, k_{\mathrm{co}}^{i}, \rho_{\mathrm{co}}^{i}\right)
$$

It is important to notice that within the first core layer, the coefficients $B_{1}$ and $D_{1}$ are zero, because $Y_{l}(x)$ is infinite at $x=0$.

\section{B. Asymptotic Approximation in the Cladding Region}

As shown in Fig. 2, the cladding region consists of two types of alternating dielectric layers, type I with refractive index $n_{\mathrm{cl}}^{1}$ and thickness $l_{\mathrm{cl}}^{1}$, and type II with refractive index $n_{\mathrm{cl}}^{2}$ and thickness $l_{\mathrm{cl}}^{2}$.

Many of the results of our original asymptotic analysis [9] can be directly carried over and applied to the fields in the cladding region. The key results are summarized in this section, whereas readers interested in more details should consult [9]. We begin with the asymptotic expression for field distribution in type I layer of the $n$th cladding pairs, as shown in (10) at the bottom of the page. Similarly, fields inside type II of the same cladding pair are given by (11), shown at the bottom of the page. In (10) and (11), $k_{\mathrm{cl}}^{1}=\sqrt{\left(n_{\mathrm{cl}}^{1} \omega / c\right)^{2}-\beta^{2}}, k_{\mathrm{cl}}^{2}=\sqrt{\left(n_{\mathrm{cl}}^{2} \omega / c\right)^{2}-\beta^{2}}$, $\rho_{\mathrm{cl}}^{n}$, and $\rho_{\mathrm{cl}}^{\prime n}$ are defined in Fig. 2. It should be noted that the TM component (including $E_{z}$ and $H_{\theta}$ ) and TE component (including $H_{z}$ and $E_{\theta}$ ) are decoupled in the asymptotic limit, with

$$
M\left(n_{\mathrm{co}}^{i}, k_{\mathrm{co}}^{i}, r\right)=\left[\begin{array}{cccc}
J_{l}\left(k_{\mathrm{cO}}^{i} r\right) & Y_{l}\left(k_{\mathrm{cO}}^{i} r\right) & 0 & 0 \\
\frac{\omega \epsilon_{0}\left(n_{\mathrm{co}}^{i}\right)^{2}}{k_{\mathrm{co}}^{i} \beta} J_{l}^{\prime}\left(k_{\mathrm{co}}^{i} r\right) & \frac{\omega \epsilon_{0}\left(n_{\mathrm{co}}^{i}\right)^{2}}{k_{\mathrm{co}}^{i} \beta} Y_{l}^{\prime}\left(k_{\mathrm{co}}^{i} r\right) & \frac{l}{\left(k_{\mathrm{co}}^{i}\right)^{2} r} J_{l}\left(k_{\mathrm{co}}^{i} r\right) & \frac{l}{\left(k_{\mathrm{c}}^{i}\right)^{2} r} Y_{l}\left(k_{\mathrm{co}}^{i} r\right) \\
0 & 0 & J_{l}\left(k_{\mathrm{co}}^{i} r\right) & Y_{l}\left(k_{\mathrm{co}}^{i} r\right) \\
\frac{l}{\left(k_{\mathrm{co}}^{i}\right)^{2} r} J_{l}\left(k_{\mathrm{co}}^{i} r\right) & \frac{l}{\left(k_{\mathrm{co}}^{i}\right)^{2} r} Y_{l}\left(k_{\mathrm{co}}^{i} r\right) & \frac{\omega \mu_{0}}{k_{\mathrm{c}}^{i} \beta} J_{l}^{\prime}\left(k_{\mathrm{co}}^{i} r\right) & \frac{\omega \mu_{0}}{k_{\mathrm{c}}^{i} \beta} Y_{l}^{\prime}\left(k_{\mathrm{co}}^{i} r\right)
\end{array}\right]
$$

$$
\begin{cases}E_{z}=\frac{f_{\mathrm{TM}}}{\sqrt{k_{\mathrm{cl}}^{1} r}}\left[a_{n} e^{i k_{\mathrm{cl}}^{1}\left(r-\rho_{\mathrm{cl}}^{n}\right)}+b_{n} e^{-i k_{\mathrm{cl}}^{1}\left(r-\rho_{\mathrm{cl}}^{n}\right)}\right] & \\ H_{\theta}=-\frac{\omega \epsilon_{0}\left(n_{\mathrm{cl}}^{1}\right)^{2}}{k_{\mathrm{cl}}^{1}} \frac{f_{\mathrm{TM}}}{\sqrt{k_{\mathrm{cl}}^{1} r}}\left[a_{n} e^{i k_{\mathrm{cl}}^{1}\left(r-\rho_{\mathrm{cl}}^{n}\right)}-b_{n} e^{-i k_{\mathrm{cl}}^{1}\left(r-\rho_{\mathrm{cl}}^{n}\right)}\right] & \rho_{\mathrm{cl}}^{n} \leq r<\rho_{\mathrm{cl}}^{n}+l_{\mathrm{cl}}^{1} . \\ H_{z}=\frac{f_{\mathrm{TE}}}{\sqrt{k_{\mathrm{cl}}^{1} r}}\left[c_{n} e^{i k_{\mathrm{cl}}^{1}\left(r-\rho_{\mathrm{cl}}^{n}\right)}+d_{n} e^{-i k_{\mathrm{cl}}^{1}\left(r-\rho_{\mathrm{cl}}^{n}\right)}\right] & \\ E_{\theta}=\frac{\omega \mu_{0}}{k_{\mathrm{cl}}^{1}} \frac{f_{\mathrm{TE}}}{\sqrt{k_{\mathrm{cl}}^{1} r}}\left[c_{n} e^{i k_{\mathrm{cl}}^{1}\left(r-\rho_{\mathrm{cl}}^{n}\right)}-d_{n} e^{-i k_{\mathrm{cl}}^{1}\left(r-\rho_{\mathrm{cl}}^{n}\right)}\right]\end{cases}
$$

$$
\begin{cases}E_{z}=\frac{f_{\mathrm{TM}}}{\sqrt{k_{\mathrm{cl}}^{2} r}}\left[a_{n}^{\prime} e^{i k_{\mathrm{cl}}^{2}\left(r-\rho_{\mathrm{cl}}^{\prime n}\right)}+b_{n}^{\prime} e^{-i k_{\mathrm{cl}}^{2}\left(r-\rho_{\mathrm{cl}}^{\prime n}\right)}\right] & \\ H_{\theta}=-\frac{\omega \epsilon_{0}\left(n_{\mathrm{cl}}^{2}\right)^{2}}{k_{\mathrm{cl}}^{2}} \frac{f_{\mathrm{TM}}}{\sqrt{k_{\mathrm{cl}}^{2} r}}\left[a_{n}^{\prime} e^{i k_{\mathrm{cl}}^{2}\left(r-\rho_{\mathrm{cl}}^{\prime n}\right)}-b_{n}^{\prime} e^{-i k_{\mathrm{cl}}^{2}\left(r-\rho_{\mathrm{cl}}^{\prime n}\right)}\right] & \rho_{\mathrm{cl}}^{\prime n} \leq r<\rho_{\mathrm{cl}}^{\prime n}+l_{\mathrm{cl}}^{2} \\ H_{z}=\frac{f_{\mathrm{TE}}}{\sqrt{k_{\mathrm{cl}}^{2} r}}\left[c_{n}^{\prime} e^{i k_{\mathrm{cl}}^{2}\left(r-\rho_{\mathrm{cl}}^{\prime n}\right)}+d_{n}^{\prime} e^{-i k_{\mathrm{cl}}^{2}\left(r-\rho_{\mathrm{cl}}^{\prime n}\right)}\right] & \\ E_{\theta}=\frac{\omega \mu_{0}}{k_{\mathrm{cl}}^{2}} \frac{f_{\mathrm{TE}}}{\sqrt{k_{\mathrm{cl}}^{2} r}}\left[c_{n}^{\prime} e^{i k_{\mathrm{cl}}^{2}\left(r-\rho_{\mathrm{cl}}^{\prime n}\right)}-d_{n}^{\prime} e^{-i k_{\mathrm{cl}}^{2}\left(r-\rho_{\mathrm{cl}}^{\prime n}\right)}\right] & \end{cases}
$$


the TM component amplitude being $f_{\mathrm{TM}}$ and the TE component amplitude being $f_{\mathrm{TE}}$. The values of $f_{\mathrm{TE}}$ and $f_{\mathrm{TM}}$ are constant within the whole Bragg cladding region.

As can be seen from (10) and (11), one of the key consequences of the asymptotic approximation is that the solution takes the form of traveling plane waves with an extra factor $1 / \sqrt{r}$. Therefore, many properties of the cylindrically symmetric Bragg stacks resemble those of planar Bragg stacks. By matching fields at interfaces between dielectric layers, we find that $a_{n}, b_{n}, c_{n}$ and $d_{n}$ in (10) are given by

$$
\begin{aligned}
& {\left[\begin{array}{l}
a_{n} \\
b_{n}
\end{array}\right]=\left(\lambda_{\mathrm{TM}}\right)^{n-1}\left[\begin{array}{c}
B_{\mathrm{TM}} \\
\lambda_{\mathrm{TM}}-A_{\mathrm{TM}}
\end{array}\right]} \\
& {\left[\begin{array}{l}
c_{n} \\
d_{n}
\end{array}\right]=\left(\lambda_{\mathrm{TE}}\right)^{n-1}\left[\begin{array}{c}
B_{\mathrm{TE}} \\
\lambda_{\mathrm{TE}}-A_{\mathrm{TE}}
\end{array}\right]}
\end{aligned}
$$

where the parameters $\lambda_{\mathrm{TE}}, \lambda_{\mathrm{TM}}, A_{\mathrm{TE}}, B_{\mathrm{TE}}, A_{\mathrm{TM}}$, and $B_{\mathrm{TM}}$ are constants within the entire cladding region and will be defined shortly. From the above expressions, it is clear that the fields at neighbor cladding pairs are the same except an overall amplitude change of $\lambda_{\mathrm{TE}}$ or $\lambda_{\mathrm{TM}}-\mathrm{a}$ direct consequence of Bloch theorem. The field amplitudes in type II layer of the $n$th cladding pair can be easily found by applying the condition of $E_{z}, E_{\theta}, H_{z}$, and $H_{\theta}$ being continuous at $r=\rho_{\text {cl }}^{\prime n}$, which gives

$$
\begin{aligned}
& {\left[\begin{array}{l}
a_{n}^{\prime} \\
b_{n}^{\prime}
\end{array}\right]=\frac{1}{2} \sqrt{\frac{k_{\mathrm{cl}}^{2}}{k_{\mathrm{cl}}^{1}}}} \\
& \cdot\left[\begin{array}{l}
\left(1+\frac{\left(n_{\mathrm{cl}}^{1}\right)^{2} k_{\mathrm{cl}}^{2}}{\left(n_{\mathrm{cl}}^{2}\right)^{2} k_{\mathrm{cl}}^{1}}\right) e^{i k_{\mathrm{cl}}^{1} l_{\mathrm{cl}}^{1}}\left(1-\frac{\left(n_{\mathrm{cl}}^{1}\right)^{2} k_{\mathrm{cl}}^{2}}{\left(n_{\mathrm{cl}}^{2}\right)^{2} k_{\mathrm{cl}}^{1}}\right) e^{-i k_{\mathrm{cl}}^{1} l_{\mathrm{cl}}^{1}} \\
\left(1-\frac{\left(n_{\mathrm{cl}}^{1}\right)^{2} k_{\mathrm{cl}}^{2}}{\left(n_{\mathrm{cl}}^{2}\right)^{2} k_{\mathrm{cl}}^{1}}\right) e^{i k_{\mathrm{cl}}^{1} l_{\mathrm{cl}}^{1}}\left(1+\frac{\left(n_{\mathrm{cl}}^{1}\right)^{2} k_{\mathrm{cl}}^{2}}{\left(n_{\mathrm{cl}}^{2}\right)^{2} k_{\mathrm{cl}}^{1}}\right) e^{-i k_{\mathrm{cl}}^{1} l_{\mathrm{cl}}^{1}}
\end{array}\right] \\
& \cdot\left[\begin{array}{l}
a_{n} \\
b_{n}
\end{array}\right]
\end{aligned}
$$

for the component and

$$
\begin{aligned}
{\left[\begin{array}{l}
c_{n}^{\prime} \\
d_{n}^{\prime}
\end{array}\right]=} & \frac{1}{2} \sqrt{\frac{k_{\mathrm{cl}}^{2}}{k_{\mathrm{cl}}^{1}}} \\
& {\left[\begin{array}{ll}
\left(1+\frac{k_{\mathrm{cl}}^{2}}{k_{\mathrm{cl}}^{1}}\right) e^{i k_{\mathrm{cl}}^{1} l_{\mathrm{cl}}^{1}} & \left(1-\frac{k_{\mathrm{cl}}^{2}}{k_{\mathrm{cl}}^{1}}\right) e^{-i k_{\mathrm{cl}}^{1} l_{\mathrm{cl}}^{1}} \\
\left(1-\frac{k_{\mathrm{cl}}^{2}}{k_{\mathrm{cl}}^{1}}\right) e^{i k_{\mathrm{cl}}^{1} l_{\mathrm{cl}}^{1}} & \left(1+\frac{k_{\mathrm{cl}}^{2}}{k_{\mathrm{cl}}^{1}}\right) e^{-i k_{\mathrm{cl}}^{1} l_{\mathrm{cl}}^{1}}
\end{array}\right] } \\
& \cdot\left[\begin{array}{l}
c_{n} \\
b_{n}
\end{array}\right]
\end{aligned}
$$

for the TE component.
As in [9], the parameters $A_{\mathrm{TE}}, B_{\mathrm{TE}}, A_{\mathrm{TM}}, B_{\mathrm{TM}}, \lambda_{\mathrm{TE}}$, and $\lambda_{\mathrm{TM}}$ in (12) and (13) are defined as

$$
\begin{aligned}
& A_{\mathrm{TE}}=e^{i k_{\mathrm{cl}}^{1} l_{\mathrm{cl}}^{1}}\left[i \frac{\left(k_{\mathrm{cl}}^{1}\right)^{2}+\left(k_{\mathrm{cl}}^{2}\right)^{2}}{2 k_{\mathrm{cl}}^{1} k_{\mathrm{cl}}^{2}}\right. \\
& \left.\cdot \sin \left(k_{\mathrm{cl}}^{2} l_{\mathrm{cl}}^{2}\right)+\cos \left(k_{\mathrm{cl}}^{2} l_{\mathrm{cl}}^{2}\right)\right]
\end{aligned}
$$

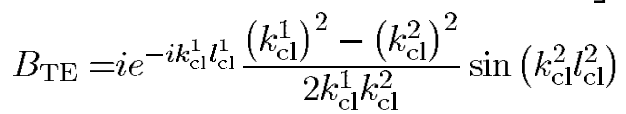

$$
\begin{aligned}
& A_{\mathrm{TM}}=e^{i k_{\mathrm{cl}}^{1} l_{\mathrm{cl}}^{1}}\left[i \frac{\left(n_{\mathrm{cl}}^{2}\right)^{4}\left(k_{\mathrm{cl}}^{1}\right)^{2}+\left(n_{\mathrm{cl}}^{1}\right)^{4}\left(k_{\mathrm{cl}}^{2}\right)^{2}}{2\left(n_{\mathrm{cl}}^{1}\right)^{2}\left(n_{\mathrm{cl}}^{2}\right)^{2} k_{\mathrm{cl}}^{1} k_{\mathrm{cl}}^{2}}\right. \\
& \left.\cdot \sin \left(k_{\mathrm{cl}}^{2} l_{\mathrm{cl}}^{2}\right)+\cos \left(k_{\mathrm{cl}}^{2} l_{\mathrm{cl}}^{2}\right)\right] \\
& B_{\mathrm{TM}}=i e^{-i k_{\mathrm{cl}}^{1} l_{\mathrm{cl}}^{1} \frac{\left(n_{\mathrm{cl}}^{2}\right)^{4}\left(k_{\mathrm{cl}}^{1}\right)^{2}-\left(n_{\mathrm{cl}}^{1}\right)^{4}\left(k_{\mathrm{cl}}^{2}\right)^{2}}{2\left(n_{\mathrm{cl}}^{1}\right)^{2}\left(n_{\mathrm{cl}}^{2}\right)^{2} k_{\mathrm{cl}}^{1} k_{\mathrm{cl}}^{2}}} \\
& \cdot \sin \left(k_{\mathrm{cl}}^{2} l_{\mathrm{cl}}^{2}\right) \\
& \lambda_{\mathrm{TE}}=\operatorname{Re}\left(A_{\mathrm{TE}}\right) \pm \sqrt{\left[\operatorname{Re}\left(A_{\mathrm{TE}}\right)\right]^{2}-1} \\
& \lambda_{\mathrm{TM}}=\operatorname{Re}\left(A_{\mathrm{TM}}\right) \pm \sqrt{\left[\operatorname{Re}\left(A_{\mathrm{TM}}\right)\right]^{2}-1} .
\end{aligned}
$$

We notice that there are two solutions for $\lambda_{\mathrm{TE}}$ and $\lambda_{\mathrm{TM}}$. In the Bragg bandgap, the two solutions of $\lambda_{\mathrm{TE}}$ and $\lambda_{\mathrm{TM}}$ are real numbers, with one having absolute value less than one and the other greater than one. We shall take the solutions of $\lambda_{\mathrm{TE}}$ and $\lambda_{\mathrm{TM}}$ with absolute values less than unity, because they correspond to modes decaying in the Bragg cladding.

Once we find the values of $a_{n}, b_{n}, c_{n}, d_{n}, a_{n}^{\prime}, b_{n}^{\prime}, c_{n}^{\prime}$, and $d_{n}^{\prime}$ by combining (12) through (21), the only unknown quantities in (10) and (11), which give the electromagnetic field in the entire cladding region, are $f_{\mathrm{TE}}$ and $f_{\mathrm{TM}}$, the amplitudes of the TE and TM components. The problem of finding them lies at the center of our asymptotic matrix formalism and will be treated in detail in Section II-C.

\section{Matrix Formalism}

The guided modes in a Bragg fiber are founded by matching the exact solution in the core region [i.e., (6)] with the asymptotic solution in the cladding region (i.e., (10)) at the interface $r=\rho_{\mathrm{Co}}^{N}=\rho_{\mathrm{cl}}^{1}$ (see Fig. 2), which gives us (22), shown at the bottom of the page. We then relate the amplitude coefficients in the $N$ th core layer (i.e., $A_{N}, B_{N}, C_{N}$, and $D_{N}$ ) to the coefficients in the first core layer (i.e., $A_{1}, B_{1}, C_{1}$, and $D_{1}$ ). We

$$
M\left(n_{\mathrm{co}}^{N}, k_{\mathrm{co}}^{N}, \rho_{\mathrm{co}}^{N}\right)\left[\begin{array}{c}
A_{N} \\
B_{N} \\
C_{N} \\
D_{N}
\end{array}\right]=\left[\begin{array}{c}
\frac{f_{\mathrm{TM}}}{\sqrt{k_{\mathrm{cl}}^{1} \rho_{\mathrm{cl}}^{1}}}\left(\lambda_{\mathrm{TM}}-A_{\mathrm{TM}}+B_{\mathrm{TM}}\right) \\
-\frac{i \omega \epsilon_{0}\left(n_{\mathrm{cl}}^{1}\right)^{2}}{k_{\mathrm{cl}}^{1} \beta} \frac{f_{\mathrm{TM}}}{\sqrt{k_{\mathrm{c}}^{1}} \rho_{\mathrm{cl}}^{1}}\left(\lambda_{\mathrm{TM}}-A_{\mathrm{TM}}-B_{\mathrm{TM}}\right) \\
\frac{f_{\mathrm{TE}}}{\sqrt{k_{\mathrm{cl}}^{1} \rho_{\mathrm{cl}}^{1}}}\left(\lambda_{\mathrm{TE}}-A_{\mathrm{TE}}+B_{\mathrm{TE}}\right) \\
-\frac{i \omega \mu_{0}}{k_{\mathrm{cl}}^{1} \beta} \frac{f_{\mathrm{TE}}}{\sqrt{k_{\mathrm{cl}}^{1} \rho_{\mathrm{cl}}^{1}}}\left(\lambda_{\mathrm{TE}}-A_{\mathrm{TE}}-B_{\mathrm{TE}}\right)
\end{array}\right] .
$$


remember that in the first core layer $B_{1}=D_{1}=0$ and we further denote $A_{1}$ as $\mathcal{A}_{\mathrm{TM}}$ and $C_{1}$ as $\mathcal{C}_{\mathrm{TE}}$. Applying (8) and (9) repeatedly, we have

$$
\begin{aligned}
{\left[\begin{array}{l}
A_{N} \\
B_{N} \\
C_{N} \\
D_{N}
\end{array}\right]=} & \mathbf{T}_{\mathrm{N}-1} \cdots \mathbf{T}_{\mathbf{2}}\left[M\left(n_{\mathrm{co}}^{2}, k_{\mathrm{co}}^{2}, \rho_{\mathrm{co}}^{1}\right)\right]^{-1} \\
& {\left[\begin{array}{cc}
J_{l}\left(k_{\mathrm{co}}^{1} \rho_{\mathrm{co}}^{1}\right) & 0 \\
\frac{\omega \epsilon_{0}\left(n_{\mathrm{co}}^{1}\right)^{2}}{k_{\mathrm{co}}^{1} \beta} J_{l}^{\prime}\left(k_{\mathrm{co}}^{1} \rho_{\mathrm{co}}^{1}\right) & \frac{l}{\left(k_{\mathrm{co}}^{1}\right)^{2} \rho_{\mathrm{c}}^{1}} J_{l}\left(k_{\mathrm{co}}^{1} \rho_{\mathrm{co}}^{1}\right) \\
0 & J_{l}\left(k_{\mathrm{co}}^{1} \rho_{\mathrm{co}}^{1}\right) \\
\frac{l}{\left(k_{\mathrm{co}}^{1}\right)^{2} \rho_{\mathrm{co}}^{1}} J_{l}\left(k_{\mathrm{co}}^{1} \rho_{\mathrm{co}}^{1}\right) & \frac{\omega \mu_{0}}{k_{\mathrm{co}}^{1} \beta} J_{l}^{\prime}\left(k_{\mathrm{co}}^{1} \rho_{\mathrm{co}}^{1}\right)
\end{array}\right] } \\
& \cdot\left[\begin{array}{c}
\mathcal{A}_{\mathrm{TM}} \\
\mathcal{C}_{\mathrm{TE}}
\end{array}\right] .
\end{aligned}
$$

Substituting (23) into (22), we find the following matrix relation (24), shown at the bottom of the page, where an overall transfer matrix $\mathbf{T}$ is defined as

$$
\begin{aligned}
\mathbf{T}= & {\left[M\left(n_{\mathrm{co}}^{2}, k_{\mathrm{co}}^{2}, \rho_{\mathrm{co}}^{1}\right) M^{-1}\left(n_{\mathrm{co}}^{2}, k_{\mathrm{co}}^{2}, \rho_{\mathrm{co}}^{2}\right)\right] } \\
& \cdots\left[M\left(n_{\mathrm{co}}^{N}, k_{\mathrm{co}}^{N}, \rho_{\mathrm{co}}^{N-1}\right) M^{-1}\left(n_{\mathrm{co}}^{N}, k_{\mathrm{co}}^{N}, \rho_{\mathrm{co}}^{N}\right)\right] \\
= & \prod_{i=2}^{N}\left[M\left(n_{\mathrm{co}}^{i}, k_{\mathrm{co}}^{i}, \rho_{\mathrm{co}}^{i-1}\right) M^{-1}\left(n_{\mathrm{co}}^{i}, k_{\mathrm{CO}}^{i}, \rho_{\mathrm{co}}^{i}\right)\right] \\
= & {\left[\begin{array}{llll}
t_{11} & t_{12} & t_{13} & t_{14} \\
t_{21} & t_{22} & t_{23} & t_{24} \\
t_{31} & t_{32} & t_{33} & t_{34} \\
t_{41} & t_{42} & t_{43} & t_{44}
\end{array}\right] . }
\end{aligned}
$$

In (24), $\mathcal{A}_{\mathrm{TM}}$ and $\mathcal{C}_{\mathrm{TE}}$, which represent the field in the first core layer, are linearly related to field in the first cladding layer ( $f_{\mathrm{TM}}$ and $f_{\mathrm{TE}}$ ) via a $4 \times 4$ transfer matrix $\mathbf{T}$ as defined in (25). Equation (24) gives us four equations with four independent variables, $\mathcal{A}_{\mathrm{TM}}, \mathcal{C}_{\mathrm{TE}}, f_{\mathrm{TM}}$, and $f_{\mathrm{TE}}$, and is sufficient to determine the propagation constant $\beta$ and field distribution of guided
Bragg fiber modes. To see this more clearly, we introduce eight new parameters $g_{\mathrm{TM}}^{j}$ and $g_{\mathrm{TE}}^{j}, j=1, \ldots, 4$ as

$$
\begin{aligned}
& g_{\mathrm{TE}}^{j}= t_{j 3}\left(\lambda_{\mathrm{TE}}-A_{\mathrm{TE}}+B_{\mathrm{TE}}\right) \\
&-\frac{i \omega \mu_{0}}{k_{\mathrm{cl}}^{1} \beta} t_{j 4}\left(\lambda_{\mathrm{TE}}-A_{\mathrm{TE}}-B_{\mathrm{TE}}\right), \\
& g_{\mathrm{TM}}^{j}= t_{j 1}\left(\lambda_{\mathrm{TM}}-A_{\mathrm{TM}}+B_{\mathrm{TM}}\right) \\
&-\frac{i \omega \epsilon_{0}\left(n_{\mathrm{cl}}^{1}\right)^{2}}{k_{\mathrm{cl}}^{1} \beta} t_{j 2}\left(\lambda_{\mathrm{TM}}-A_{\mathrm{TM}}-B_{\mathrm{TM}}\right), \\
& j=1, \ldots, 4
\end{aligned}
$$

where $t_{j 1}, j_{j 2}, t_{j 3}$, and $t_{j 4}$ are the matrix elements given in (25). With these new parameters, we can split (24) into (28) and (29), shown at the bottom of the page. These two equations lie at the center of our asymptotic matrix method. To fully understand their consequences, we consider two separate cases, the TE or TM modes with $l=0$ and the mixed modes with $l \neq 0$.

The TE or TM modes, in contrast with the doubly degenerate $l \neq 0$ modes, are truly single mode and, therefore, are of special interest. If a Bragg fiber is designed such that it supports only a single TE or TM mode, many undesirable polarization-dependent phenomena can be completely eliminated. For modes with $l=0$, we first notice that the matrix $M\left(n_{\mathrm{cO}}^{i}, k_{\mathrm{co}}^{i}, r\right)$ is block diagonalized into two $2 \times 2$ matrices. As a result, the transfer matrix $\mathbf{T}$, as defined in (25), is also block diagonalized into two $2 \times 2$ matrices with $t_{31}=t_{41}=t_{32}=t_{42}=t_{13}=t_{23}=$ $t_{14}=t_{24}=0$. According to the definitions in (26) and (27), we have $g_{\mathrm{TM}}^{3}=g_{\mathrm{TM}}^{4}=0$ and $g_{\mathrm{TE}}^{1}=g_{\mathrm{TE}}^{2}=0$.

By definition, the $H_{z}$ component of any TM mode must remain zero in the entire Bragg fiber, i.e., $\mathcal{C}_{\mathrm{TE}}=0$ and $f_{\mathrm{TE}}=0$. With this condition in mind, from (28), we can easily find

$$
\frac{\omega \epsilon_{0}\left(n_{\mathrm{co}}^{1}\right)^{2}}{k_{\mathrm{CO}}^{1} \beta} \frac{J_{0}^{\prime}\left(k_{\mathrm{co}}^{1} \rho_{\mathrm{co}}^{1}\right)}{J_{0}\left(k_{\mathrm{CO}}^{1} \rho_{\mathrm{CO}}^{1}\right)}=\frac{g_{\mathrm{TM}}^{2}}{g_{\mathrm{TM}}^{1}} .
$$

Once we have specified the Bragg fiber parameters and chosen the frequency $\omega$, the propagation constants of TM modes are

$$
\left[\begin{array}{cc}
J_{l}\left(k_{\mathrm{co}}^{1} \rho_{\mathrm{co}}^{1}\right) & 0 \\
\frac{\omega \epsilon_{0}\left(n_{\mathrm{co}}^{1}\right)^{2}}{k_{\mathrm{co}}^{1} \beta} J_{l}^{\prime}\left(k_{\mathrm{co}}^{1} \rho_{\mathrm{co}}^{1}\right) & \frac{l}{\left(k_{\mathrm{co}}^{1}\right)^{2} \rho_{\mathrm{co}}^{1}} J_{l}\left(k_{\mathrm{co}}^{1} \rho_{\mathrm{co}}^{1}\right) \\
0 & J_{l}\left(k_{\mathrm{co}}^{1} \rho_{\mathrm{co}}^{1}\right) \\
\frac{l}{\left(k_{\mathrm{co}}^{1}\right)^{2} \rho_{\mathrm{co}}^{1}} J_{l}\left(k_{\mathrm{co}}^{1} \rho_{\mathrm{co}}^{1}\right) & \frac{\omega \mu_{0}}{k_{\mathrm{co}}^{1} \beta} J_{l}^{\prime}\left(k_{\mathrm{co}}^{1} \rho_{\mathrm{co}}^{1}\right)
\end{array}\right]\left[\begin{array}{c}
\mathcal{A}_{\mathrm{TM}} \\
\mathcal{C}_{\mathrm{TE}}
\end{array}\right]=\mathbf{T}\left[\begin{array}{c}
\frac{f_{\mathrm{TM}}}{\sqrt{k_{\mathrm{cl}}^{1} \rho_{\mathrm{cl}}^{1}}}\left(\lambda_{\mathrm{TM}}-A_{\mathrm{TM}}+B_{\mathrm{TM}}\right) \\
-\frac{i \omega \epsilon_{0}\left(n_{\mathrm{cl}}^{1}\right)^{2}}{k_{\mathrm{cl}}^{1} \beta} \frac{f_{\mathrm{TM}}}{\sqrt{k_{\mathrm{c}}^{1} \rho_{\mathrm{cl}}^{1}}}\left(\lambda_{\mathrm{TM}}-A_{\mathrm{TM}}-B_{\mathrm{TM}}\right) \\
\frac{f_{\mathrm{TE}}}{\sqrt{k_{\mathrm{cl}}^{1} \rho_{\mathrm{cl}}^{1}}}\left(\lambda_{\mathrm{TE}}-A_{\mathrm{TE}}+B_{\mathrm{TE}}\right) \\
-\frac{i \omega \mu_{0}}{k_{\mathrm{cl}}^{1} \beta} \frac{f_{\mathrm{TE}}}{\sqrt{k_{\mathrm{cl}}^{1} \rho_{\mathrm{cl}}^{1}}}\left(\lambda_{\mathrm{TE}}-A_{\mathrm{TE}}-B_{\mathrm{TE}}\right)
\end{array}\right]
$$

$$
\begin{aligned}
& {\left[\begin{array}{cc}
J_{l}\left(k_{\mathrm{co}}^{1} \rho_{\mathrm{co}}^{1}\right) & 0 \\
\frac{\omega \epsilon_{0}\left(n_{\mathrm{co}}^{1}\right)^{2}}{k_{\mathrm{co}}^{1} \beta} J_{l}^{\prime}\left(k_{\mathrm{co}}^{1} \rho_{\mathrm{co}}^{1}\right) & \frac{l}{\left(k_{\mathrm{co}}^{1}\right)^{2} \rho_{\mathrm{co}}^{1}} J_{l}\left(k_{\mathrm{co}}^{1} \rho_{\mathrm{co}}^{1}\right)
\end{array}\right]\left[\begin{array}{l}
\mathcal{A}_{\mathrm{TM}} \\
\mathcal{C}_{\mathrm{TE}}
\end{array}\right]=\frac{1}{\sqrt{k_{\mathrm{cl}}^{1} \rho_{\mathrm{cl}}^{1}}}\left[\begin{array}{ll}
g_{\mathrm{TM}}^{1} & g_{\mathrm{TE}}^{1} \\
g_{\mathrm{TM}}^{2} & g_{\mathrm{TE}}^{2}
\end{array}\right]\left[\begin{array}{l}
f_{\mathrm{TM}} \\
f_{\mathrm{TE}}
\end{array}\right]} \\
& {\left[\begin{array}{cc}
0 & J_{l}\left(k_{\mathrm{co}}^{1} \rho_{\mathrm{co}}^{1}\right) \\
\frac{l}{\left(k_{\mathrm{co}}^{1}\right)^{2} \rho_{\mathrm{co}}^{1}} J_{l}\left(k_{\mathrm{co}}^{1} \rho_{\mathrm{co}}^{1}\right) & \frac{\omega \mu_{0}}{k_{\mathrm{co}}^{1} \beta} J_{l}^{\prime}\left(k_{\mathrm{co}}^{1} \rho_{\mathrm{co}}^{1}\right)
\end{array}\right]\left[\begin{array}{l}
\mathcal{A}_{\mathrm{TM}} \\
\mathcal{C}_{\mathrm{TE}}
\end{array}\right]=\frac{1}{\sqrt{k_{\mathrm{cl}}^{1} \rho_{\mathrm{cl}}^{1}}}\left[\begin{array}{ll}
g_{\mathrm{TM}}^{3} & g_{\mathrm{TE}}^{3} \\
g_{\mathrm{TM}}^{4} & g_{\mathrm{TE}}^{4}
\end{array}\right]\left[\begin{array}{l}
f_{\mathrm{TM}} \\
f_{\mathrm{TE}}
\end{array}\right] .}
\end{aligned}
$$


found by solving for $\beta_{\mathrm{TM}}$ satisfying (30). We substitute the result $\beta_{\mathrm{TM}}$ back into (28), and obtain the following relation:

$$
\mathcal{A}_{\mathrm{TM}}=\frac{g_{\mathrm{TM}}^{1}}{J_{0}\left(k_{\mathrm{CO}}^{1} \rho_{\mathrm{CO}}^{1}\right) \sqrt{k_{\mathrm{cl}}^{1} \rho_{\mathrm{cl}}^{1}}} f_{\mathrm{TM}} .
$$

The importance of this result is that it relates the mode amplitude $\mathcal{A}_{\mathrm{TM}}$ in the first core layer to $f_{\mathrm{TM}}$, which, as discussed in Section II-B, determines the fields within the entire fiber cladding region. We can choose the normalization factor of the guided mode such that $\mathcal{A}_{\mathrm{TM}}=1$. Combining this condition with (31), $f_{\mathrm{TE}}=0$ and (10) through (21) in Section II-B, we obtain the TM field distribution in the cladding region. The TM field distribution in the core region can also be easily found. In the center core layer, we have $A_{1}=\mathcal{A}_{\mathrm{TM}}=1$ and $B_{1}=C_{1}=D_{1}=$ 0 . Applying (8) repeatedly, where the transfer matrices $\mathbf{T}_{\mathbf{i}}$ are found from (7) and (9), we find all the mode coefficients $A_{i}$, $B_{i}, C_{i}$, and $D_{i}$ in the $N$ core layers. The TM field distribution in the core region is simply given by substituting these mode coefficients into (6) and applying (7).

For TE modes, we have $A_{\mathrm{TM}}=0$, and (29) gives us

$$
\begin{aligned}
\frac{\omega \mu_{0}}{k_{\mathrm{CO}}^{1} \beta} \frac{J_{0}^{\prime}\left(k_{\mathrm{co}}^{1} \rho_{\mathrm{co}}^{1}\right)}{J_{0}\left(k_{\mathrm{co}}^{1} \rho_{\mathrm{co}}^{1}\right)} & =\frac{g_{\mathrm{TE}}^{4}}{g_{\mathrm{TE}}^{3}} \\
\mathcal{C}_{\mathrm{TE}} & =\frac{g_{\mathrm{TE}}^{3}}{J_{0}\left(k_{\mathrm{co}}^{1} \rho_{\mathrm{co}}^{1}\right) \sqrt{k_{\mathrm{cl}}^{1} \rho_{\mathrm{cl}}^{1}}} f_{\mathrm{TE}} .
\end{aligned}
$$

Following the same procedure as for TM modes, we can find the propagation constant $\beta$ and field distribution for TE modes from (32) and (33).

For any Bragg fiber mode with $l \neq 0$, both (28) and (29) are needed and the solutions are more complicated. To simplify our final results, we introduce more definitions

$$
\begin{aligned}
H_{\mathrm{TE}}^{1}= & -J_{l}\left(k_{\mathrm{CO}}^{1} \rho_{\mathrm{co}}^{1}\right) g_{\mathrm{TE}}^{4}+\frac{\omega \mu_{0}}{k_{\mathrm{CO}}^{1} \beta} J_{l}^{\prime}\left(k_{\mathrm{co}}^{1} \rho_{\mathrm{co}}^{1}\right) g_{\mathrm{TE}}^{3} \\
& +\frac{l}{\left(k_{\mathrm{co}}^{1}\right)^{2} \rho_{\mathrm{co}}^{1}} J_{l}\left(k_{\mathrm{co}}^{1} \rho_{\mathrm{co}}^{1}\right) g_{\mathrm{TE}}^{1} \\
H_{\mathrm{TE}}^{2}= & J_{l}\left(k_{\mathrm{co}}^{1} \rho_{\mathrm{co}}^{1}\right) g_{\mathrm{TE}}^{2}-\frac{\omega \epsilon_{0}\left(n_{\mathrm{co}}^{1}\right)^{2}}{k_{\mathrm{co}}^{1} \beta} J_{l}^{\prime}\left(k_{\mathrm{co}}^{1} \rho_{\mathrm{co}}^{1}\right) g_{\mathrm{TE}}^{1} \\
& -\frac{l}{\left(k_{\mathrm{co}}^{1}\right)^{2} \rho_{\mathrm{co}}^{1}} J_{l}\left(k_{\mathrm{co}}^{1} \rho_{\mathrm{co}}^{1}\right) g_{\mathrm{TE}}^{3} \\
H_{\mathrm{TM}}^{1}= & J_{l}\left(k_{\mathrm{co}}^{1} \rho_{\mathrm{co}}^{1}\right) g_{\mathrm{TM}}^{4}-\frac{\omega \mu_{0}}{k_{\mathrm{co}}^{1} \beta} J_{l}^{1}\left(k_{\mathrm{co}}^{1} \rho_{\mathrm{co}}^{1}\right) g_{\mathrm{TM}}^{3} \\
& -\frac{l}{\left(k_{\mathrm{co}}^{1}\right)^{2} \rho_{\mathrm{co}}^{1}} J_{l}\left(k_{\mathrm{co}}^{1} \rho_{\mathrm{co}}^{1}\right) g_{\mathrm{TM}}^{1} \\
H_{\mathrm{TM}}^{2}= & -J_{l}\left(k_{\mathrm{co}}^{1} \rho_{\mathrm{co}}^{1}\right) g_{\mathrm{TM}}^{2}+\frac{\omega \epsilon_{0}\left(n_{\mathrm{co}}^{1}\right)^{2}}{k_{\mathrm{CO}}^{1} \beta} J_{l}^{\prime}\left(k_{\mathrm{co}}^{1} \rho_{\mathrm{co}}^{1}\right) g_{\mathrm{TM}}^{1} \\
& +\frac{l}{\left(k_{\mathrm{co}}^{1}\right)^{2} \rho_{\mathrm{co}}^{1}} J_{l}\left(k_{\mathrm{co}}^{1} \rho_{\mathrm{co}}^{1}\right) g_{\mathrm{TM}}^{3} .
\end{aligned}
$$

To find the propagation constant $\beta$ of any mixed mode, we first express $\mathcal{A}_{\mathrm{TM}}$ and $\mathcal{C}_{\mathrm{TE}}$ in terms of $f_{\mathrm{TM}}$ and $f_{\mathrm{TE}}$ by in- verting the leftmost $2 \times 2$ matrix in (28). Substituting the results of $\mathcal{A}_{\mathrm{TM}}$ and $\mathcal{C}_{\mathrm{TE}}$ into (29), we find

$$
\left[\begin{array}{ll}
H_{\mathrm{TM}}^{1} & -H_{\mathrm{TE}}^{1} \\
H_{\mathrm{TM}}^{2} & -H_{\mathrm{TE}}^{2}
\end{array}\right]\left[\begin{array}{l}
f_{\mathrm{TM}} \\
f_{\mathrm{TE}}
\end{array}\right]=0
$$

with $H_{\mathrm{TE}}^{1}, H_{\mathrm{TE}}^{2}, H_{\mathrm{TM}}^{1}$, and $H_{\mathrm{TM}}^{2}$ defined in (34)-(37). In order for (38) to have nonzero solutions, the determinant of the matrix must be zero, which gives

$$
\frac{H_{\mathrm{TM}}^{1}}{H_{\mathrm{TM}}^{2}}=\frac{H_{\mathrm{TE}}^{1}}{H_{\mathrm{TE}}^{2}} .
$$

As can be seen from the definitions in (34)-(37), (25)-(27), and (7), the parameters $H_{\mathrm{TE}}^{1}, H_{\mathrm{TE}}^{2}, H_{\mathrm{TM}}^{1}$, and $H_{\mathrm{TM}}^{2}$ are complicated. However, once the Bragg fiber structure is chosen and the frequency is given, they only depend on $\beta$. Therefore, the solution of (39) gives us the propagation constant of any mixed mode.

After finding the solutions of (39) and choosing an appropriate normalization constant, we can determine the values of $f_{\mathrm{TM}}$ and $f_{\mathrm{TE}}$ from (38) as

$$
\left[\begin{array}{l}
f_{\mathrm{TM}} \\
f_{\mathrm{TE}}
\end{array}\right]=\frac{l}{\left(k_{\mathrm{co}}^{1}\right)^{2} \rho_{\mathrm{co}}^{1}}\left[J_{l}\left(k_{\mathrm{co}}^{1} \rho_{\mathrm{co}}^{1}\right)\right]^{2} \sqrt{k_{\mathrm{cl}}^{1} \rho_{\mathrm{cl}}^{1}}\left[\begin{array}{l}
H_{\mathrm{TE}}^{1} \\
H_{\mathrm{TM}}^{1}
\end{array}\right] .
$$

As before, by combining this result with (10) to (21) in Section II-B, we can find the whole cladding field distribution. To obtain the fields in the fiber core region, we substitute (40) into (29) and find (41), shown at the bottom of the page. Thus, within the first core layer, we have $A_{1}=\mathcal{A}_{\mathrm{TM}}, C_{1}=\mathcal{C}_{\mathrm{TE}}$, and $B_{1}=D_{1}=0$. By applying (6)-(9) in Section II-A throughout the entire core layers, we find the electromagnetic fields in the Bragg fiber core region.

Comparing the method outlined in this section with our original asymptotic approach [9], the present method has several important advantages. In our original approach, the fiber core region contains only a single dielectric layer, which prevents us from analyzing more complicated Bragg fiber geometries, such as the dielectric coaxial fibers [6]. There is no such limitation in our current approach, because we can include an arbitrary number of dielectric layers in the core region. Equally important, the fields inside the core region are exact solutions of Maxwell equations, with no approximation involved. Thus, the current approach can reach an arbitrary level of precision simply by including more and more dielectric layers into the core region. As a result, the current method can still be accurate while the original approach may fail to generate satisfactory results.

\section{Surface Modes}

In the previous sections, we assume that general solutions in any layer are given by $J_{l}(x)$ and $Y_{l}(x)$, which only holds if $\beta \leq n_{\mathrm{co}}^{i} \omega / c$ for all of the core layers. In an air core Bragg fiber, a guided mode may have $\beta$ exceeding $\omega / c$, yet satisfying

$$
\left[\begin{array}{l}
\mathcal{A}_{\mathrm{TM}} \\
\mathcal{C}_{\mathrm{TE}}
\end{array}\right]=\left[\begin{array}{c}
-\frac{\omega \mu_{0}}{k_{\mathrm{co}}^{1} \beta} J_{l}^{\prime}\left(k_{\mathrm{co}}^{1} \rho_{\mathrm{co}}^{1}\right)\left(g_{\mathrm{TM}}^{3} H_{\mathrm{TE}}^{1}+g_{\mathrm{TE}}^{3} H_{\mathrm{TM}}^{1}\right)+J_{l}\left(k_{\mathrm{co}}^{1} \rho_{\mathrm{co}}^{1}\right)\left(g_{\mathrm{TM}}^{4} H_{\mathrm{TE}}^{1}+g_{\mathrm{TE}}^{4} H_{\mathrm{TM}}^{1}\right) \\
\frac{l}{\left(k_{\mathrm{co}}^{1}\right)^{2} \rho_{\mathrm{co}}^{1}} J_{l}\left(k_{\mathrm{CO}}^{1} \rho_{\mathrm{CO}}^{1}\right)\left(g_{\mathrm{TM}}^{3} H_{\mathrm{TE}}^{1}+g_{\mathrm{TE}}^{3} H_{\mathrm{TM}}^{1}\right)
\end{array}\right] .
$$


$\beta \leq n_{\mathrm{cl}}^{1} \omega / c$ and $\beta \leq n_{\mathrm{cl}}^{2} \omega / c$. Such a mode is generally referred to as the surface mode, because it decays both in the cladding layers, due to Bragg reflection, and in the air core, due to total internal reflection. In this case, we can still apply the asymptotic approximation to the cladding field and use results in Section II-B. However, in the core region, there are some important differences between regular guided modes (i.e., results in Sections II-A and II-C) and surface modes, which shall be summarized in this section.

For the $i$ th Bragg fiber layer in which $\beta>n_{\mathrm{co}}^{i} \omega / c$, the solutions of $E_{z}$ and $H_{z}$ are given by $I_{l}\left(k_{\mathrm{co}}^{i} r\right)$ and $K_{l}\left(k_{\mathrm{CO}}^{i} r\right)$ [10], with $k_{\mathrm{CO}}^{i}$ defined as

$$
k_{\mathrm{co}}^{i}=\sqrt{\beta^{2}-\left(\frac{n_{\mathrm{co}}^{i} \omega}{c}\right)^{2}} .
$$

We still use four parameters, $A_{i}, B_{i}, C_{i}$, and $D_{i}$ to express field components in the $i$ th layer, as in (6). However, the matrix $M$ has a new definition, (43), shown at the bottom of the page. If we use this new definition for $M\left(n_{\mathrm{co}}^{i}, k_{\mathrm{co}}^{i}, r\right)$ when $\beta>n_{\mathrm{co}}^{i} \omega / c$, the overall transfer matrix $\mathbf{T}$ is still the same as in (25), and $g_{\mathrm{TE}}^{j}$ and $g_{\mathrm{TM}}^{j}$ are still given by (26) and (27), respectively. If, at the center, low index core $\beta>n_{\mathrm{co}}^{1} \omega / c$, (28) and (29), which determine modal dispersion and field distribution, are replaced by (44) and (45), shown at the bottom of the page. From (44) and (45), for TM modes, we find

$$
\begin{aligned}
-\frac{\omega \epsilon_{\mathrm{O}}\left(n_{\mathrm{co}}^{1}\right)^{2}}{k_{\mathrm{cO}}^{1} \beta} \frac{I_{0}^{\prime}\left(k_{\mathrm{co}}^{1} \rho_{\mathrm{co}}^{1}\right)}{I_{0}\left(k_{\mathrm{co}}^{1} \rho_{\mathrm{cO}}^{1}\right)} & =\frac{g_{\mathrm{TM}}^{2}}{g_{\mathrm{TM}}^{1}} \\
\mathcal{A}_{\mathrm{TM}} & =\frac{g_{\mathrm{TM}}^{1}}{I_{0}\left(k_{\mathrm{c} \rho}^{1} \rho_{\mathrm{cO}}^{1}\right) \sqrt{k_{\mathrm{cl}}^{1} \rho_{\mathrm{cl}}^{1}}} f_{\mathrm{TM}}
\end{aligned}
$$

which, respectively, give dispersion and field distribution of guided TM modes. The corresponding equations for TE modes are

$$
\begin{aligned}
-\frac{\omega \mu_{0}}{k_{\mathrm{CO}}^{1} \beta} \frac{I_{0}^{\prime}\left(k_{\mathrm{co}}^{1} \rho_{\mathrm{co}}^{1}\right)}{I_{0}\left(k_{\mathrm{co}}^{1} \rho_{\mathrm{CO}}^{1}\right)} & =\frac{g_{\mathrm{TE}}^{4}}{g_{\mathrm{TE}}^{3}} \\
\mathcal{C}_{\mathrm{TE}} & =\frac{g_{\mathrm{TE}}^{3}}{I_{0}\left(k_{\mathrm{co}}^{1} \rho_{\mathrm{cO}}^{1}\right) \sqrt{k_{\mathrm{cl}}^{1} \rho_{\mathrm{cl}}^{1}}} f_{\mathrm{TE}} .
\end{aligned}
$$

The results for the mixed surface modes are more complicated. First, if $\beta>n_{\mathrm{co}}^{1} \omega / c$, the definitions of $H_{\mathrm{TE}}^{1}, H_{\mathrm{TE}}^{2}, H_{\mathrm{TM}}^{1}$, and $H_{\mathrm{TM}}^{2}$ must be changed accordingly

$$
\begin{aligned}
H_{\mathrm{TE}}^{1}= & -I_{l}\left(k_{\mathrm{co}}^{1} \rho_{\mathrm{co}}^{1}\right) g_{\mathrm{TE}}^{4}-\frac{\omega \mu_{0}}{k_{\mathrm{co}}^{1} \beta} I_{l}^{\prime}\left(k_{\mathrm{co}}^{1} \rho_{\mathrm{CO}}^{1}\right) g_{\mathrm{TE}}^{3} \\
& -\frac{l}{\left(k_{\mathrm{co}}^{1}\right)^{2} \rho_{\mathrm{co}}^{1}} I_{l}\left(k_{\mathrm{co} \rho}^{1} \rho_{\mathrm{co}}^{1}\right) g_{\mathrm{TE}}^{1} \\
H_{\mathrm{TE}}^{2}= & I_{l}\left(k_{\mathrm{co}}^{1} \rho_{\mathrm{co}}^{1}\right) g_{\mathrm{TE}}^{2}+\frac{\omega \epsilon_{0}\left(n_{\mathrm{co}}^{1}\right)^{2}}{k_{\mathrm{co}}^{1} \beta} I_{l}^{\prime}\left(k_{\mathrm{co}}^{1} \rho_{\mathrm{co}}^{1}\right) g_{\mathrm{TE}}^{1} \\
& +\frac{l}{\left(k_{\mathrm{co}}^{1}\right)^{2} \rho_{\mathrm{co}}^{1}} I_{l}\left(k_{\mathrm{co}}^{1} \rho_{\mathrm{co}}^{1}\right) g_{\mathrm{TE}}^{3} \\
H_{\mathrm{TM}}^{1}= & I_{l}\left(k_{\mathrm{co}}^{1} \rho_{\mathrm{co}}^{1}\right) g_{\mathrm{TM}}^{4}+\frac{\omega \mu_{0}}{k_{\mathrm{co}}^{1} \beta} I_{l}^{\prime}\left(k_{\mathrm{co}}^{1} \rho_{\mathrm{co}}^{1}\right) g_{\mathrm{TM}}^{3} \\
& +\frac{l}{\left(k_{\mathrm{co}}^{1}\right)^{2} \rho_{\mathrm{co}}^{1}} I_{l}\left(k_{\mathrm{co}}^{1} \rho_{\mathrm{co}}^{1}\right) g_{\mathrm{TM}}^{1} \\
H_{\mathrm{TM}}^{2}= & -I_{l}\left(k_{\mathrm{co}}^{1} \rho_{\mathrm{co}}^{1}\right) g_{\mathrm{TM}}^{2}-\frac{\omega \epsilon_{0}\left(n_{\mathrm{co}}^{1}\right)^{2}}{k_{\mathrm{co}}^{1} \beta} I_{l}^{\prime}\left(k_{\mathrm{co}}^{1} \rho_{\mathrm{co}}^{1}\right) g_{\mathrm{TM}}^{1} \\
& -\frac{l}{\left(k_{\mathrm{co}}^{1}\right)^{2} \rho_{\mathrm{co}}^{1}} I_{l}\left(k_{\mathrm{co}}^{1} \rho_{\mathrm{co}}^{1}\right) g_{\mathrm{TM}}^{3} \cdot
\end{aligned}
$$

With the new definitions, (39) still holds for surface modes and its solutions give the modal dispersion. However, the expressions for cladding field coefficients and core field coefficients are different and are, respectively, given by (54) and (55), shown at the bottom of the next page.

\section{RADIATION LOSS}

For an air core Bragg fiber, the propagation loss is the sum of two terms, the radiation loss due to the finite number of cladding pairs and the absorption loss due to the cladding materials. The absorption loss depends on the choice of cladding media and is not considered in this section. The radiation loss depends mostly on the index contrast of the cladding media and the number of cladding pairs. In this section, we use the asymptotic theory to estimate the number of cladding pairs needed to reduce the radiation loss below $0.2 \mathrm{~dB} / \mathrm{km}$.

To simplify our analysis, we study a Bragg fiber with a central air core bounded by $N$ pairs of cladding layers, as shown in

$$
M\left(n_{\mathrm{co}}^{i}, k_{\mathrm{co}}^{i}, r\right)=\left[\begin{array}{cccc}
I_{l}\left(k_{\mathrm{cO}}^{i} r\right) & K_{l}\left(k_{\mathrm{co}}^{i} r\right) & 0 & 0 \\
-\frac{\omega \epsilon_{0}\left(n_{\mathrm{co}}^{i}\right)^{2}}{k_{\mathrm{co}}^{i} \beta} I_{l}^{\prime}\left(k_{\mathrm{co}}^{i} r\right) & -\frac{\omega \epsilon_{0}\left(n_{\mathrm{co}}^{i}\right)^{2}}{k_{\mathrm{co}}^{i} \beta} K_{l}^{\prime}\left(k_{\mathrm{co}}^{i} r\right) & -\frac{l}{\left(k_{\mathrm{c}}^{i}\right)^{2} r} I_{l}\left(k_{\mathrm{co}}^{i} r\right) & -\frac{l}{\left(k_{\mathrm{co}}^{i}\right)^{2} r} K_{l}\left(k_{\mathrm{co}}^{i} r\right) \\
0 & 0 & I_{l}\left(k_{\mathrm{co}}^{i} r\right) & K_{l}\left(k_{\mathrm{co}}^{i} r\right) \\
-\frac{l}{\left(k_{\mathrm{co}}^{i}\right)^{2} r} I_{l}\left(k_{\mathrm{co}}^{i} r\right) & -\frac{l}{\left(k_{\mathrm{co}}^{i}\right)^{2} r} K_{l}\left(k_{\mathrm{co}}^{i} r\right) & -\frac{\omega \mu_{0}}{k_{\mathrm{co}}^{i} \beta} I_{l}^{\prime}\left(k_{\mathrm{co}}^{i} r\right) & -\frac{\omega \mu_{0}}{k_{\mathrm{co}}^{i} \beta} K_{l}^{\prime}\left(k_{\mathrm{co}}^{i} r\right)
\end{array}\right] .
$$

$$
\begin{aligned}
& {\left[\begin{array}{cc}
I_{l}\left(k_{\mathrm{c}}^{1} \rho_{\mathrm{co}}^{1}\right) & 0 \\
-\frac{\omega \epsilon_{0}\left(n_{\mathrm{c} \rho}^{1}\right)^{2}}{k_{\mathrm{co}}^{1} \beta} I_{l}^{\prime}\left(k_{\mathrm{co}}^{1} \rho_{\mathrm{co}}^{1}\right) & -\frac{l}{\left(k_{\mathrm{co}}^{1}\right)^{2} \rho_{\mathrm{co}}^{1}} I_{l}\left(k_{\mathrm{co}}^{1} \rho_{\mathrm{co}}^{1}\right)
\end{array}\right]\left[\begin{array}{l}
\mathcal{A}_{\mathrm{TM}} \\
\mathcal{C}_{\mathrm{TE}}
\end{array}\right]=\frac{1}{\sqrt{k_{\mathrm{cl}}^{1} \rho_{\mathrm{cl}}^{1}}}\left[\begin{array}{ll}
g_{\mathrm{TM}}^{1} & g_{\mathrm{TE}}^{1} \\
g_{\mathrm{TM}}^{2} & g_{\mathrm{TE}}^{2}
\end{array}\right]\left[\begin{array}{l}
f_{\mathrm{TM}} \\
f_{\mathrm{TE}}
\end{array}\right]} \\
& {\left[\begin{array}{cc}
0 & I_{l}\left(k_{\mathrm{co}}^{1} \rho_{\mathrm{co}}^{1}\right) \\
-\frac{l}{\left(k_{\mathrm{co}}^{1}\right)^{2} \rho_{\mathrm{co}}^{1}} I_{l}\left(k_{\mathrm{co}}^{1} \rho_{\mathrm{co}}^{1}\right) & -\frac{\omega \mu_{0}}{k_{\mathrm{co}}^{1} \beta} I_{l}^{\prime}\left(k_{\mathrm{co}}^{1} \rho_{\mathrm{co}}^{1}\right)
\end{array}\right]\left[\begin{array}{l}
\mathcal{A}_{\mathrm{TM}} \\
\mathcal{C}_{\mathrm{TE}}
\end{array}\right]=\frac{1}{\sqrt{k_{\mathrm{cl}}^{1} \rho_{\mathrm{cl}}^{1}}}\left[\begin{array}{ll}
g_{\mathrm{TM}}^{3} & g_{\mathrm{TE}}^{3} \\
g_{\mathrm{TM}}^{4} & g_{\mathrm{TE}}^{4}
\end{array}\right]\left[\begin{array}{l}
f_{\mathrm{TM}} \\
f_{\mathrm{TE}}
\end{array}\right] \text {. }}
\end{aligned}
$$




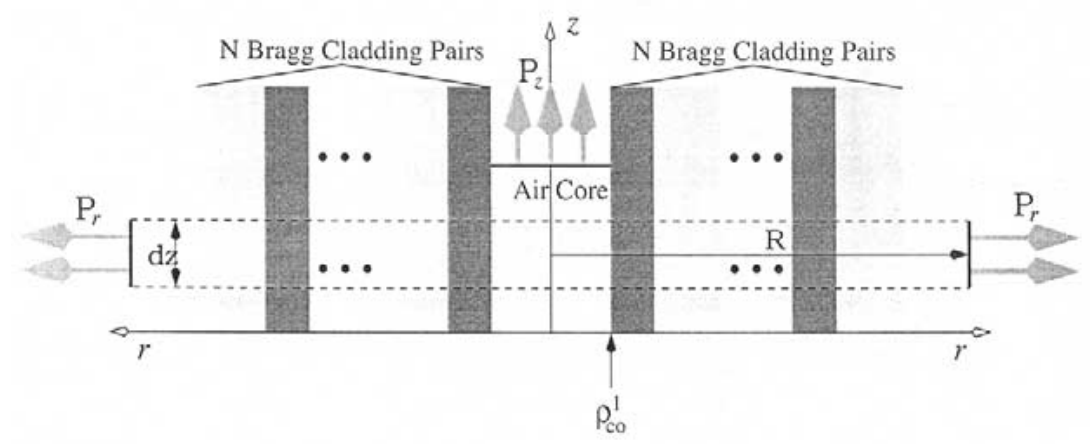

Fig. 3. The radiation loss of an air core Bragg fiber with $N$ Bragg cladding pairs. The figure show the $r-z$ cross section of the Bragg fiber. $P_{z}$, the power flux inside the air core, is the integration of the Poynting vector over the cross section of the fiber air core. The radial power flux $P_{r}$ is given by integrating the Poynting vector over a cylindrical surface with radius $R$ and height $d z$, as shown.

Fig. 3. We treat the air core exactly and apply the asymptotic approximation to the entire Bragg cladding structure. As a direct result, the transfer matrix $\mathbf{T}$ that relates the cladding region to the core region is simply a $4 \times 4$ identity matrix whose diagonal terms are one and the off-diagonal terms are zero. Following (26) and (27), we find

$$
\begin{aligned}
& g_{\mathrm{TE}}^{3}=\lambda_{\mathrm{TE}}-A_{\mathrm{TE}}+B_{\mathrm{TE}} \\
& g_{\mathrm{TE}}^{4}=-\frac{i \omega \mu_{0}}{k_{\mathrm{cl}}^{1} \beta}\left(\lambda_{\mathrm{TE}}-A_{\mathrm{TE}}-B_{\mathrm{TE}}\right) \\
& g_{\mathrm{TM}}^{1}=\lambda_{\mathrm{TM}}-A_{\mathrm{TM}}+B_{\mathrm{TM}} \\
& g_{\mathrm{TM}}^{2}=-\frac{i \omega \epsilon_{0}\left(n_{\mathrm{cl}}^{1}\right)^{2}}{k_{\mathrm{cl}}^{1} \beta}\left(\lambda_{\mathrm{TM}}-A_{\mathrm{TM}}-B_{\mathrm{TM}}\right) .
\end{aligned}
$$

Other values of $g_{\mathrm{TE}}^{i}$ and $g_{\mathrm{TM}}^{i}$ are all zero. As a further simplification, we shall confine ourselves to the study of TE and TM modes. The reasons are twofold. First, as we have mentioned before, the modes with $l=0$ are of special interest, because they do not have any polarization dependent effects. Second, in the asymptotic limit, the mixed modes $(l \neq 0)$ in the cladding structure can always be classified into TE component and TM components. Therefore, we can expect the radiation loss of mixed modes to exhibit characteristics between those of TE and TM modes.
We first consider TE modes. According to (6), the $H_{z}(r)$ component in the Bragg fiber core is simply $H_{z}(r)=$ $\mathcal{C}_{\mathrm{TE}} J_{0}\left(k_{\mathrm{co}}^{1} r\right)$ and the other two components are [1]

$$
\begin{aligned}
& E_{\theta}=-i \frac{\omega \mu_{0}}{k_{\mathrm{co}}^{1}} \mathcal{C}_{\mathrm{TE}} J_{0}^{\prime}\left(k_{\mathrm{co}}^{1} r\right) \\
& H_{r}=\frac{i \beta}{k_{\mathrm{co}}^{1}} \mathcal{C}_{\mathrm{TE}} J_{0}^{\prime}\left(k_{\mathrm{CO}}^{1} r\right) .
\end{aligned}
$$

From these expressions for $E_{\theta}$ and $H_{r}$, we find the power flux along the $z$ direction in the low index core

$$
P_{z}^{\mathrm{TE}}=\left|\mathcal{C}_{\mathrm{TE}}\right|^{2} \frac{\pi \omega \mu_{0} \beta}{\left(k_{\mathrm{CO}}^{1}\right)^{2}} \int_{0}^{\rho_{\mathrm{co}}^{1}} d r r\left[J_{0}^{\prime}\left(k_{\mathrm{co}}^{1} r\right)\right]^{2} .
$$

If the Bragg fiber consists of an infinite number of cladding pairs, the asymptotic fields in the $(N+1)$ th cladding pair can be extracted from (10) as (63) and (64), shown at the bottom of the page. In (63) and (64), we notice that the fields consist two components, an outgoing wave with amplitude proportional to $c_{N+1}$ and an incoming wave with amplitude proportional to $d_{N+1}$. It can be shown that the two components are of equal value and cancel each other such that the Bragg fiber has no net radial power flux. This, however, is directly due to the assumption of infinite cladding pairs. In fact, we can regard the incoming component in the $N+1$ th cladding pair as due to the reflection by the remaining Bragg cladding. Therefore, it is reasonable to assume that if the Bragg fiber has only $N$ cladding

$$
\begin{aligned}
{\left[\begin{array}{l}
f_{\mathrm{TM}} \\
f_{\mathrm{TE}}
\end{array}\right] } & =\frac{l}{\left(k_{\mathrm{co}}^{1}\right)^{2} \rho_{\mathrm{co}}^{1}}\left[I_{l}\left(k_{\mathrm{co}}^{1} \rho_{\mathrm{co}}^{1}\right)\right]^{2} \sqrt{k_{\mathrm{cl}}^{1} \rho_{\mathrm{cl}}^{1}}\left[\begin{array}{l}
H_{\mathrm{TE}}^{1} \\
H_{\mathrm{TM}}^{1}
\end{array}\right] \\
{\left[\begin{array}{l}
\mathcal{A}_{\mathrm{TM}} \\
\mathcal{C}_{\mathrm{TE}}
\end{array}\right] } & =\left[\begin{array}{c}
-\frac{\omega \mu_{0}}{k_{\mathrm{co}}^{1} \beta} I_{l}^{\prime}\left(k_{\mathrm{co}}^{1} \rho_{\mathrm{co}}^{1}\right)\left(g_{\mathrm{TM}}^{3} H_{\mathrm{TE}}^{1}+g_{\mathrm{TE}}^{3} H_{\mathrm{TM}}^{1}\right)-I_{l}\left(k_{\mathrm{CO}}^{1} \rho_{\mathrm{CO}}^{1}\right)\left(g_{\mathrm{TM}}^{4} H_{\mathrm{TE}}^{1}+g_{\mathrm{TE}}^{4} H_{\mathrm{TM}}^{1}\right) \\
\frac{l}{\left(k_{\mathrm{co}}^{1}\right)^{2} \rho_{\mathrm{co}}^{1}} I_{l}\left(k_{\mathrm{co}}^{1} \rho_{\mathrm{CO}}^{1}\right)\left(g_{\mathrm{TM}}^{3} H_{\mathrm{TE}}^{1}+g_{\mathrm{TE}}^{3} H_{\mathrm{TM}}^{1}\right)
\end{array}\right] .
\end{aligned}
$$

$$
\begin{aligned}
& H_{z}=\frac{f_{\mathrm{TE}}}{\sqrt{k_{\mathrm{cl}}^{1} r}}\left[c_{N+1} e^{i k_{\mathrm{cl}}^{1}\left(r-\rho_{\mathrm{cl}}^{N+1}\right)}+d_{N+1} e^{-i k_{\mathrm{cl}}^{1}\left(r-\rho_{\mathrm{cl}}^{N+1}\right)}\right] \\
& E_{\theta}=\frac{\omega \mu_{0}}{k_{\mathrm{cl}}^{1}} \frac{f_{\mathrm{TE}}}{\sqrt{k_{\mathrm{cl}}^{1} r}}\left[c_{N+1} e^{i k_{\mathrm{cl}}^{1}\left(r-\rho_{\mathrm{cl}}^{N+1}\right)}-d_{N+1} e^{-i k_{\mathrm{cl}}^{1}\left(r-\rho_{\mathrm{cl}}^{N+1}\right)}\right] .
\end{aligned}
$$


pairs, the radiation field outside the cladding structure can be well approximated by the outgoing component of (63) and (64), i.e., we replace $d_{N+1}$ with 0 . To calculate the radial power flow, let us consider a cylindrical surface with radius $R$ and height $d z$ that encloses the Bragg fiber, as shown in Fig. 3. Using (63) and (64), and taking $d_{N+1}=0$, we find the radial power flux through this surface to be

$$
P_{r}^{\mathrm{TE}}=\frac{\pi \omega \mu_{0}}{\left(k_{\mathrm{cl}}^{1}\right)^{2}}\left|f_{\mathrm{TE}}\right|^{2}\left|c_{N+1}\right|^{2} d z .
$$

For TE modes propagating along the $z$ direction of the Bragg fiber, with the presence of radiation loss, the optical power decays as $\exp \left(-\alpha_{\mathrm{TE}} z\right)$. The parameter $\alpha_{\mathrm{TE}}$ is the radiation loss constant and, from the definitions of $P_{z}^{\mathrm{TE}}$ and $P_{r}^{\mathrm{TE}}$, we can identify $\alpha_{\mathrm{TE}}$ as

$$
\begin{aligned}
\alpha_{\mathrm{TE}}= & \frac{P_{r}^{\mathrm{TE}}}{P_{z}^{\mathrm{TE}} d z} \\
= & \frac{1}{\beta}\left(\frac{k_{\mathrm{co}}^{1}}{k_{\mathrm{cl}}^{1}}\right)^{2}\left|\frac{B_{\mathrm{TE}}}{\lambda_{\mathrm{TE}}-A_{\mathrm{TE}}+B_{\mathrm{TE}}}\right|^{2}\left|\lambda_{\mathrm{TE}}\right|^{2 N} \\
& \cdot \frac{\left[J_{0}\left(k_{\mathrm{co}}^{1} \rho_{\mathrm{co}}^{1}\right)\right]^{2} k_{\mathrm{cl}}^{1} \rho_{\mathrm{cl}}^{1}}{\int_{0}^{\rho_{\mathrm{co}}^{1}} d r r\left[J_{0}^{\prime}\left(k_{\mathrm{CO}}^{1} r\right)\right]^{2}}
\end{aligned}
$$

where (13), (33), (56), (62), and (65) are used.

For TM modes, we can follow the same procedure to obtain the radiation loss constant $\alpha_{\mathrm{TM}}$. First, we find fields in the low index core and the corresponding power flux in $z$ direction

$$
\begin{aligned}
H_{\theta} & =\frac{i \omega \epsilon_{0}\left(n_{\mathrm{co}}^{1}\right)^{2}}{k_{\mathrm{CO}}^{1}} \mathcal{A}_{\mathrm{TM}} J_{0}^{\prime}\left(k_{\mathrm{co}}^{1} r\right) \\
E_{r} & =\frac{i \beta}{k_{\mathrm{co}}^{1}} \mathcal{A}_{\mathrm{TM}} J_{0}^{\prime}\left(k_{\mathrm{co}}^{1} r\right) \\
P_{z}^{\mathrm{TM}} & =\left|\mathcal{A}_{\mathrm{TM}}\right|^{2} \frac{\pi \omega \epsilon_{0}\left(n_{\mathrm{co}}^{1}\right)^{2} \beta}{\left(k_{\mathrm{co}}^{1}\right)^{2}} \int_{0}^{\rho_{\mathrm{co}}^{1}} d r r\left[J_{0}^{\prime}\left(k_{\mathrm{co}}^{1} r\right)\right]^{2} .
\end{aligned}
$$

The outgoing radiation field outside the $N$ th cladding pairs can also be identified from (10) as

$$
\begin{aligned}
& E_{z}=\frac{f_{\mathrm{TM}}}{\sqrt{k_{\mathrm{cl}}^{1} r}} a_{N+1} e^{i k_{\mathrm{cl}}^{1}\left(r-\rho_{\mathrm{cl}}^{N+1}\right)} \\
& H_{\theta}=-\frac{\omega \epsilon_{0}\left(n_{\mathrm{cl}}^{1}\right)^{2}}{k_{\mathrm{cl}}^{1}} \frac{f_{\mathrm{TM}}}{\sqrt{k_{\mathrm{cl}}^{1} r}} a_{N+1} e^{i k_{\mathrm{cl}}^{1}\left(r-\rho_{\mathrm{cl}}^{N+1}\right)}
\end{aligned}
$$

and, correspondingly, the radial power flux is

$$
P_{r}^{\mathrm{TM}}=\frac{\pi \omega \epsilon_{0}\left(n_{\mathrm{cl}}^{1}\right)^{2}}{\left(k_{\mathrm{cl}}^{1}\right)^{2}}\left|f_{\mathrm{TM}}\right|^{2}\left|a_{N+1}\right|^{2} d z
$$

which gives the radiation loss constant to be

$$
\begin{aligned}
\alpha_{\mathrm{TM}}= & \frac{P_{r}^{\mathrm{TM}}}{P_{z}^{\mathrm{TM}} d z} \\
= & \frac{1}{\beta}\left(\frac{n_{\mathrm{cl}}^{1} k_{\mathrm{co}}^{1}}{n_{\mathrm{co}}^{1} k_{\mathrm{cl}}^{1}}\right)^{2}\left|\frac{B_{\mathrm{TM}}}{\lambda_{\mathrm{TM}}-A_{\mathrm{TM}}+B_{\mathrm{TM}}}\right|^{2}\left|\lambda_{\mathrm{TM}}\right|^{2 N} \\
& \times \frac{\left[J_{0}\left(k_{\mathrm{co}}^{1} \rho_{\mathrm{co}}^{1}\right)\right]^{2} k_{\mathrm{cl}}^{1} \rho_{\mathrm{cl}}^{1}}{\int_{0}^{\rho_{\mathrm{co}}^{1}} d r r\left[J_{0}^{\prime}\left(k_{\mathrm{co}}^{1} r\right)\right]^{2}}
\end{aligned}
$$

where (12), (31), (58), (69), and (72) are used.
To simplify our results for $\alpha_{\mathrm{TE}}$ and $\alpha_{\mathrm{TM}}$, we introduce a new parameter $x=k_{\mathrm{co}}^{1} \rho_{\mathrm{co}}^{1}$. For the fiber structure shown in Fig. 3, $\rho_{\mathrm{cl}}^{1}$ is the same as $\rho_{\mathrm{co}}^{1}$, which gives

$$
\frac{\left[J_{0}\left(k_{\mathrm{co}}^{1} \rho_{\mathrm{co}}^{1}\right)\right]^{2} k_{\mathrm{cl}}^{1} \rho_{\mathrm{cl}}^{1}}{\int_{0}^{\rho_{\mathrm{co}}^{1}} d r r\left[J_{0}^{\prime}\left(k_{\mathrm{co}}^{1} r\right)\right]^{2}}=k_{\mathrm{co}}^{1} k_{\mathrm{cl}}^{1} \frac{x\left[J_{0}(x)\right]^{2}}{\int_{0}^{x} d u u\left[J_{1}(u)\right]^{2}}
$$

where we have applied $J_{0}^{\prime}(x)=-J_{1}(x)$ [10]. The exact value of this expression depends on our choice of $x$. However, for an order-of-magnitude estimate, we can simply choose $x=3.8317$, the first zero point of $J_{1}(x)$, and the integral $\int_{0}^{x} d u \quad u\left[J_{1}(u)\right]^{2}$ becomes $x^{2}\left[J_{2}(x)\right]^{2} / 2$ (see, for example, [10]). Combining these results, we find

$$
\frac{\left[J_{0}\left(k_{\mathrm{co}}^{1} \rho_{\mathrm{co}}^{1}\right)\right]^{2} k_{\mathrm{cl}}^{1} \rho_{\mathrm{cl}}^{1}}{\int_{0}^{\rho_{\mathrm{co}}^{1}} d r r\left[J_{0}^{\prime}\left(k_{\mathrm{co}}^{1} r\right)\right]^{2}} \approx 0.522 k_{\mathrm{co}}^{1} k_{\mathrm{cl}}^{1}
$$

As can be seen from (16) to (21), $A_{\mathrm{TE}}, B_{\mathrm{TE}}, \lambda_{\mathrm{TE}}, A_{\mathrm{TM}}$, $B_{\mathrm{TM}}$, and $\lambda_{\mathrm{TM}}$ have the same order of magnitude. Therefore, in our estimation of radiation loss, we take the values of $B_{\mathrm{TE}} /\left(\lambda_{\mathrm{TE}}-A_{\mathrm{TE}}+B_{\mathrm{TE}}\right)$ and $B_{\mathrm{TM}} /\left(\lambda_{\mathrm{TM}}-A_{\mathrm{TM}}+B_{\mathrm{TM}}\right)$ to be 1 . Combining these approximations with (75), we find that (66) and (73) become

$$
\begin{aligned}
\alpha_{\mathrm{TE}} & =0.522 \frac{\left(k_{\mathrm{co}}^{1}\right)^{3}}{\beta k_{\mathrm{cl}}^{1}}\left|\lambda_{\mathrm{TE}}\right|^{2 N} \\
\alpha_{\mathrm{TM}} & =0.522\left(\frac{n_{\mathrm{cl}}^{1}}{n_{\mathrm{co}}^{1}}\right)^{2} \frac{\left(k_{\mathrm{co}}^{1}\right)^{3}}{\beta k_{\mathrm{cl}}^{1}}\left|\lambda_{\mathrm{TM}}\right|^{2 N} .
\end{aligned}
$$

These two expression can be further simplified by taking $n_{\mathrm{co}}^{1}=$ 1 (air core), $\lambda=2 \pi c / \omega=1.55 \mu \mathrm{m}$, and assuming $\beta=k_{\mathrm{co}}^{1}=$ $\omega / \sqrt{2} c, k_{\mathrm{cl}}^{1}=n_{\mathrm{cl}}^{1} \omega / c$

$$
\begin{aligned}
& \alpha_{\mathrm{TE}}(\mathrm{dB} / \mathrm{km})=4.6 \times 10^{9} \frac{1}{n_{\mathrm{cl}}^{1}}\left|\lambda_{\mathrm{TE}}\right|^{2 N} \\
& \alpha_{\mathrm{TM}}(\mathrm{dB} / \mathrm{km})=4.6 \times 10^{9} n_{\mathrm{cl}}^{1}\left|\lambda_{\mathrm{TM}}\right|^{2 N}
\end{aligned}
$$

where the unit for radiation loss has been converted to decibels per kilometer.

Many assumptions are made to simplify (66) and (73) into (78) and (79). It is worthwhile to see how can we justify the simplified results from an intuitive point of view. Without the Bragg cladding, the light confinement can only be achieved on the order of the wavelength, which means that the radiation loss constant must be of the order of $\mathrm{dB} / \mu \mathrm{m}=10^{9} \mathrm{~dB} / \mathrm{km}$. With the presence of Bragg cladding, the light amplitude reduction due to each cladding pair is $\lambda_{\mathrm{TE}}$ for TE modes and $\lambda_{\mathrm{TM}}$ for TM modes. Therefore, the radiation loss for a fiber with $N$ Bragg cladding pairs should be of the order of $\left(\lambda_{\mathrm{TE}}\right)^{2 N} \times 10^{9} \mathrm{~dB} / \mathrm{km}$ for TE modes and $\left(\lambda_{\mathrm{TM}}\right)^{2 N} \times 10^{9} \mathrm{~dB} / \mathrm{km}$ for TM modes.

The values of $\lambda_{\mathrm{TE}}$ and $\lambda_{\mathrm{TM}}$ also have complicated dependence on $\beta, n_{\mathrm{cl}}^{1}, l_{\mathrm{cl}}^{1}, n_{\mathrm{cl}}^{2}$, and $l_{\mathrm{cl}}^{2}$, as can be seen from (16)-(21). However, when the cladding layers form quarter wave stack (i.e., $k_{\mathrm{cl}}^{1} l_{\mathrm{cl}}^{1}=k_{\mathrm{cl}}^{2} l_{\mathrm{cl}}^{2}=\pi / 2$ ) such that light is optimally confined, the expressions for $\left|\lambda_{\mathrm{TE}}\right|$ and $\left|\lambda_{\mathrm{TM}}\right|$ take simpler forms

$$
\begin{aligned}
& \left|\lambda_{\mathrm{TE}}\right|=\min \left(\frac{k_{\mathrm{cl}}^{2}}{k_{\mathrm{cl}}^{1}}, \frac{k_{\mathrm{cl}}^{1}}{k_{\mathrm{cl}}^{2}}\right) \\
& \left|\lambda_{\mathrm{TM}}\right|=\min \left[\left(\frac{n_{\mathrm{cl}}^{2}}{n_{\mathrm{cl}}^{1}}\right)^{2} \frac{k_{\mathrm{cl}}^{1}}{k_{\mathrm{cl}}^{2}},\left(\frac{n_{\mathrm{cl}}^{1}}{n_{\mathrm{cl}}^{2}}\right)^{2} \frac{k_{\mathrm{cl}}^{2}}{k_{\mathrm{cl}}^{1}}\right] .
\end{aligned}
$$




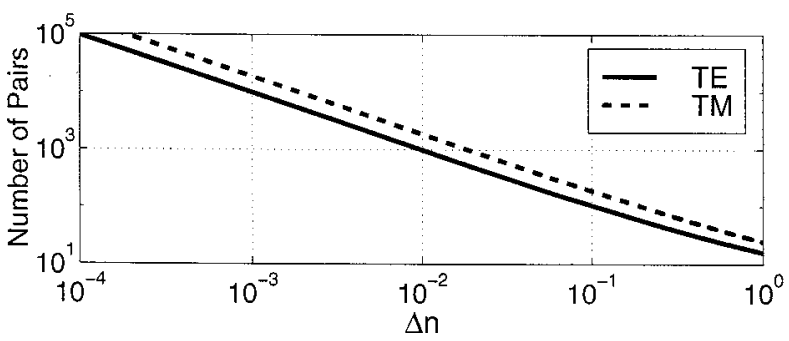

(a)

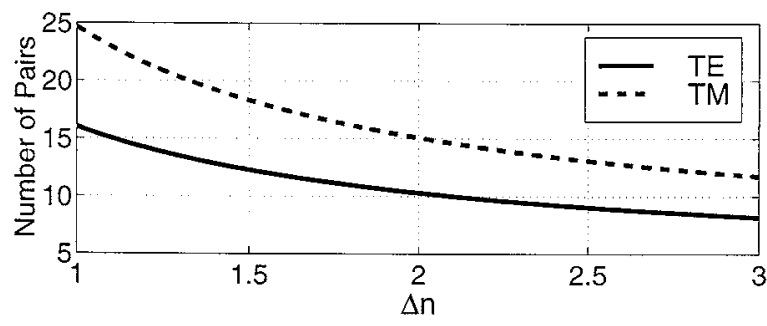

(b)

Fig. 4. The number of Bragg cladding pairs necessary to achieve $0.2-\mathrm{dB} / \mathrm{km}$ radiation loss. $\Delta n$ is defined as the index contrast between the two cladding dielectric media $n_{\mathrm{cl}}^{1}-n_{\mathrm{cl}}^{2}$. The vertical axis is the number of Bragg cladding pairs. The refractive index of the low index medium is $n_{\mathrm{cl}}^{2}=1.5$. The solid line gives the minimum number of Bragg pairs required to reduce the radiation loss of TE modes to $0.2 \mathrm{~dB} / \mathrm{km}$. The dash line gives the corresponding quantitiy for the TM modes. (a) Weak index contrast. (b) Large index contrast.

We choose cladding layer II to be the low index medium with $n_{\mathrm{cl}}^{2}=1.5$, typical of silica glass and polymers. With this value, it can be shown that for $0<\beta<\omega / c$, the minimum value of $\left|\lambda_{\mathrm{TE}}\right|$ is $\sqrt{\left[\left(n_{\mathrm{cl}}^{2}\right)^{2}-1\right] /\left[\left(n_{\mathrm{cl}}^{1}\right)^{2}-1\right]}$ and the minimum value of $\left|\lambda_{\mathrm{TM}}\right|$ is $n_{\mathrm{cl}}^{2} / n_{\mathrm{cl}}^{1}$. Substituting them into (78) and (79), we find that the minimum number of Bragg layers pairs required to achieve $0.2 \mathrm{~dB} / \mathrm{km}$ radiation loss is

$$
N_{\mathrm{TE}}=\frac{23.9-\ln \left(n_{\mathrm{cl}}^{1}\right)}{\ln \left[\left(n_{\mathrm{cl}}^{1}\right)^{2}-1\right]-\ln \left[\left(n_{\mathrm{cl}}^{2}\right)^{2}-1\right]}
$$

for TE modes and

$$
N_{\mathrm{TM}}=\frac{23.9+\ln \left(n_{\mathrm{cl}}^{1}\right)}{2\left[\ln \left(n_{\mathrm{cl}}^{1}\right)-\ln \left(n_{\mathrm{cl}}^{2}\right)\right]}
$$

for TM modes.

We plot (82) and (83) in Fig. 4. Fig. 4(a) corresponds to the case of weak index contrast. For $\Delta n$ less than 0.01 , it takes 1000 or more cladding pairs to reach $0.2 \mathrm{~dB} / \mathrm{km}$. Fabricating such a large number of cladding pairs is likely to be very difficult, in practice. For $\Delta n$ between 0.1 and 1 , we find that it takes less than 200 cladding pairs to reduce the radiation loss of TE and TM modes to $0.2 \mathrm{~dB} / \mathrm{km}$. We notice that this index contrast range corresponds to what can be achieved in the air core photonic bandgap (PBG) fiber [5]. Of course, the light confinement in PBG fibers is achieved through 2-D Bragg reflection rather than one-dimensional (1-D) Bragg reflection. However, if we take an effective index approach and approximate the 2-D air hole patterns as alternating layers of concentric dielectric layers with high and low refractive index, the index contrast between the effective refractive indexes should fall within the range of 0.1 to 1 . Thus, for air core PBG fibers, $0.2-\mathrm{dB} / \mathrm{km}$ propagation loss can be achieved with 200 or fewer air hole layers. Fig. 4(b) corresponds to the case of large index contrast. We notice that for
$\Delta n$ between 1 and $3\left(2.5<n_{\mathrm{cl}}^{1}<4.5\right), 25$ pairs may suffice to guide TE and TM modes with less than $0.2 \mathrm{~dB} / \mathrm{km}$ radiation loss.

We have only discussed radiation loss for TE and TM modes so far. According to the discussions in Section II-C, modes with $l \neq 0$ are mixtures of TE and TM components in the Bragg cladding layers. Therefore, their radiation loss is determined by the TM component, because TM component is less confined and suffers more radiation loss compared with TE component, as can be seen from Fig. 4. Thus, we can apply our results for TM modes to estimate the radiation loss of mixed modes.

Because we use the smallest possible values for $\left|\lambda_{\mathrm{TE}}\right|$ and $\left|\lambda_{\mathrm{TM}}\right|$ in deriving (82) and (83), our results in Fig. 4 give the minimum number of Bragg pairs needed to achieve $0.2-\mathrm{dB} / \mathrm{km}$ radiation loss and should serve as an order of magnitude estimate. Obtaining a better estimate of radiation loss requires values of $\beta, n_{\mathrm{cl}}^{1}, l_{\mathrm{cl}}^{1}, n_{\mathrm{cl}}^{2}$, and $l_{\mathrm{cl}}^{2}$. Once they are known, we can find $\lambda_{\mathrm{TE}}$ and $\lambda_{\mathrm{TM}}$ from (20) and (21) and substitute them into (76) and (77) for $\alpha_{\mathrm{TE}}$ and $\alpha_{\mathrm{TM}}$.

One notable exception to this estimate is worth mentioning. In an air core fiber, if $\beta$ is only slightly less than $\omega / c$, the value of $k_{\mathrm{co}}^{1}$ can be very close to zero, whereas in deriving (82) and (83), we use $\beta=k_{\text {co }}^{1}=\omega / \sqrt{2} c$. According to (66) and (73), a small $k_{\mathrm{co}}^{1}$ can greatly reduce the radiation loss [14]. Therefore, when $\beta$ becomes very close to $\omega / c$, it may be necessary to use (66) and (73) to obtain accurate results of radiation loss.

\section{RESULTS}

In this section, we use both the asymptotic theory in Section II-C and a 2-D FDTD method to analyze the modal dispersion of an air core Bragg fiber. The 2-D FDTD algorithm we use in this paper follows that in [13]. The key observation is that, for any mode propagating in a dielectric structure uniform in the $z$ direction, the $z$ dependence of the fields is simply $\exp (i \beta z)$. For example, we can write the electric field as $\vec{E}(x, y, t) \exp (i \beta z)$ and the magnetic field as $\vec{H}(x, y, t) \exp (i \beta z)$. As a result, the three-dimensional (3-D) Maxwell equations are reduced to 2-D ones

$$
\begin{aligned}
& \left(\nabla_{\perp}+i \beta \vec{e}_{z}\right) \times \vec{E}(x, y, t)=-\mu_{0} \frac{\partial}{\partial t} \vec{H}(x, y, t) \\
& \left(\nabla_{\perp}+i \beta \vec{e}_{z}\right) \times \vec{H}(x, y, t)=\epsilon_{0} \epsilon(x, y) \frac{\partial}{\partial t} \vec{E}(x, y, t)
\end{aligned}
$$

where $\nabla_{\perp}$ is defined as $(\partial / \partial x) \vec{e}_{x}+(\partial / \partial y) \vec{e}_{y}$. We can transform these 2-D differential equations into a set of finite difference equations following the standard finite difference time domain procedure [11]. At the boundaries of the computational domain, we use the perfectly matched layer boundary condition (PML) [15], [16] to absorb all of the outgoing radiation. For the 2-D FDTD equations and other details about the implementation of the algorithm, the reader should consult [13].

We first use the asymptotic algorithm outlined in Section II-C to study the dispersion properties of a Bragg fiber. The results will be compared with those obtained from 2-D FDTD calculations to verify the validity of the asymptotic approach. We choose to study an air core $\left(n_{\mathrm{co}}^{1}=1.0\right)$ Bragg fiber with cladding parameters as $n_{\mathrm{cl}}^{1}=4.6, l_{\mathrm{cl}}^{1}=0.25 \Lambda, n_{\mathrm{cl}}^{2}=1.5$, and $l_{\mathrm{cl}}^{2}=0.75 \Lambda$, where the parameters are defined in Fig. 2 and $\Lambda=l_{\mathrm{cl}}^{1}+l_{\mathrm{cl}}^{2}$ is the total thickness of a Bragg cladding pair. We 


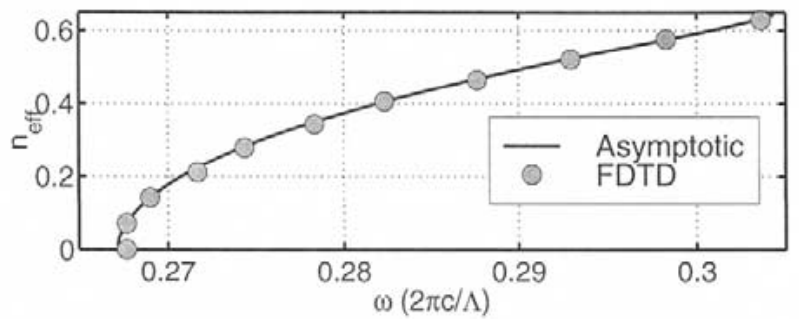

(a)

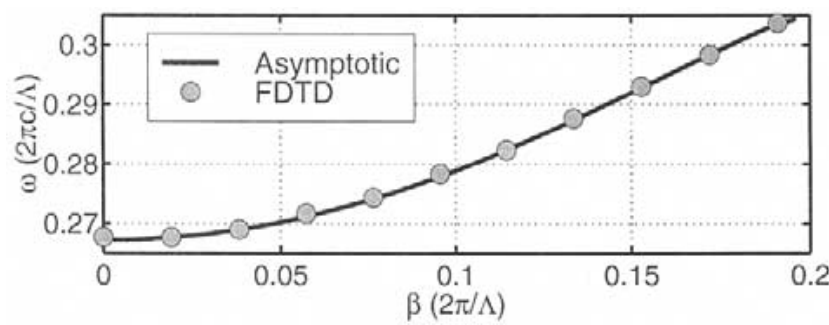

(b)

Fig. 5. The dispersion of an air core Bragg fiber with parameters $n_{\mathrm{co}}^{1}=1.0$, $\rho_{\mathrm{co}}^{1}=1.0 \Lambda, n_{\mathrm{cl}}^{1}=4.6, l_{\mathrm{cl}}^{1}=0.25 \Lambda, n_{\mathrm{cl}}^{2}=1.5$, and $l_{\mathrm{cl}}^{2}=0.75 \Lambda$. In the frequency range plotted in the figure, the Bragg fiber supports a guided mode propagating in the air core. The azimuthal mode number is $l=1$. The solid line is the dispersion relation given by the asymptotic analysis, whereas the dots represent the 2-D finite difference time domain results. The core region in our asymptotic calculations consists of five dielectric layers, the air core and the first two cladding pairs. In our FDTD calculations, we choose $\Lambda=24$ computational cells. The effective indexes $n_{\text {eff }}$ is defined as $\beta c / \omega$. (a) Effective index $n_{\text {eff }}=$ $\beta c / \omega$ as a function of $\omega$. (b) $\omega$ as a function of propagation constant $\beta$.

choose the air core radius to be $\rho_{\mathrm{co}}^{1}=1.0 \Lambda$. In the asymptotic calculations, the core region consists of five concentric dielectric layers. Using the notations in Fig. 2, we explicitly write out the core region parameters as $n_{\mathrm{co}}^{1}=1.0, n_{\mathrm{co}}^{2}=n_{\mathrm{co}}^{4}=4.6$, $n_{\mathrm{co}}^{3}=n_{\mathrm{co}}^{5}=1.5, l_{\mathrm{co}}^{1}=1.0 \Lambda, l_{\mathrm{co}}^{2}=l_{\mathrm{co}}^{4}=0.25 \Lambda$, and $l_{\mathrm{co}}^{3}=l_{\mathrm{co}}^{5}=0.75 \Lambda$. In 2-D FDTD calculations, we choose $\Lambda=24$ computational cells and use three cladding pairs around the air core to define the Bragg fiber. In Fig. 4, we find that for index contrast we have chosen, 10 cladding pairs are enough to reduce the radiation loss to approximately $0.2 \mathrm{~dB} / \mathrm{km}$. Not surprisingly, three cladding pairs should give us well-defined guided modes.

Both the asymptotic results and the FDTD results are shown in Fig. 5, where, in Fig. 5(a), we plot the effective index $n_{\mathrm{eff}}=$ $\beta c / \omega$ as a function of $\omega$ and, in Fig. 5(b), we plot $\omega$ as a function of propagation constant $\beta$. Notice that the units for $\beta$ and $\omega$ are, respectively, $2 \pi / \Lambda$ and $2 \pi c / \Lambda$. Within the frequency range shown in Fig. 5, both the asymptotic analysis and FDTD calculations show that the Bragg fiber supports a guided mode propagating in the air core. The azimuthal dependence of the mode is $\cos (\theta)$ or $\sin (\theta)(l=1)$. We notice that, in Fig. 5, the two approaches agree well with each other. The main source for the small discrepancy is the discretization error in the finite difference time domain algorithm. In fact, if we consider that only six computational cells are used for $l_{\mathrm{cl}}^{1}$, the agreement between the asymptotic approach and FDTD approach is quite impressive.

In Fig. 6, we show the distribution of the $H_{z}$ field obtained from FDTD calculation. The frequency and propagation constant of the mode are respectively $\omega=0.291(2 \pi c / \Lambda)$ and $\beta=0.143(2 \pi / \Lambda)$. Fig. 6 clearly shows that the guided mode

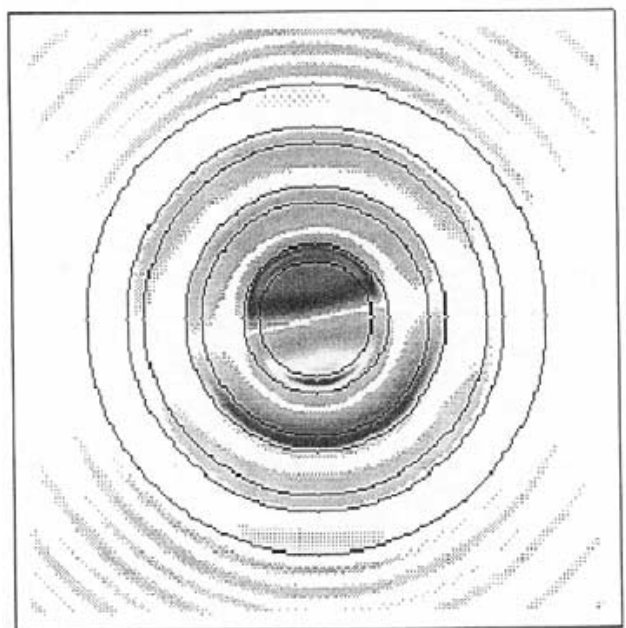

Fig. 6. The $H_{z}$ field distribution of a guided Bragg fiber mode. The parameters of the Bragg fiber are given in caption of Fig. 5. The Bragg cladding consists of only three layer pairs. The frequency and propagation constant of the mode are respectively $\omega=0.291(2 \pi c / \Lambda)$ and $\beta=0.143(2 \pi / \Lambda)$.

has an azimuthal number $l=1$ and most of the field is concentrated within the air core and the first cladding layer. The radiation field outside of the Bragg cladding can also be seen in Fig. 6.

As mentioned at the end of Section II-C, a main advantage of our new asymptotic algorithm is that the result can be arbitrarily precise by incorporating more and more dielectric layers into the core region. More specifically, if we use a superscript $N$ to denote the asymptotic results obtained using an inner core region consist of $N$ dielectric layers, the results should converge as a function of $N$ to the exact solutions. With this expectation in mind, we analyze the same Bragg fiber described in the caption of Fig. 5 and study how the results depend on the number of inner core region layers. We first choose a core region consist of seven dielectric layers (including the center air core plus three cladding pairs) and calculate the effective index of the Bragg fiber. We denote the result as $n_{\mathrm{eff}}^{7}$ and use this as the standard for comparison. Then we calculate the effective indexes using one, three, and five inner core layers and, respectively, denote the results as $n_{\mathrm{eff}}^{1}, n_{\mathrm{eff}}^{3}$, and $n_{\mathrm{eff}}^{5}$. The absolute value of the difference of between these values and $n_{\text {eff }}^{7}$ are plotted in Fig. 7, i.e., $\left|n_{\mathrm{eff}}^{1}-n_{\mathrm{eff}}^{7}\right|,\left|n_{\mathrm{eff}}^{3}-n_{\mathrm{eff}}^{7}\right|$ and $\left|n_{\mathrm{eff}}^{5}-n_{\mathrm{eff}}^{7}\right|$.

We first notice that the difference between $n_{\text {eff }}^{1}$, where the core region consists of only the air core and the standard $n_{\mathrm{eff}}^{7}$, is quite large. In fact, as frequency approaches $0.265(2 \pi / \Lambda)$, the cutoff frequency of the guided mode, the difference between the two values reaches 0.2 , which is quite significant. On the other hand, with the addition of only one cladding pair into the core region, the deviation between the effective indexes $\left(\left|n_{\text {eff }}^{3}-n_{\text {eff }}^{7}\right|\right)$ is reduced below 0.02 , a $10 \times$ reduction compared with $\left|n_{\text {eff }}^{1}-n_{\text {eff }}^{7}\right|$. Because one more cladding pair added into the core region, $\left|n_{\mathrm{eff}}^{5}-n_{\mathrm{eff}}^{7}\right|$ is below 0.001 . This suggests that the difference between asymptotic results and exact solutions should also be of the same order of magnitude. Thus, we can conclude that asymptotic results with inner core region consist of the first five dielectric layers offer an excellent approximation of the exact solution. 


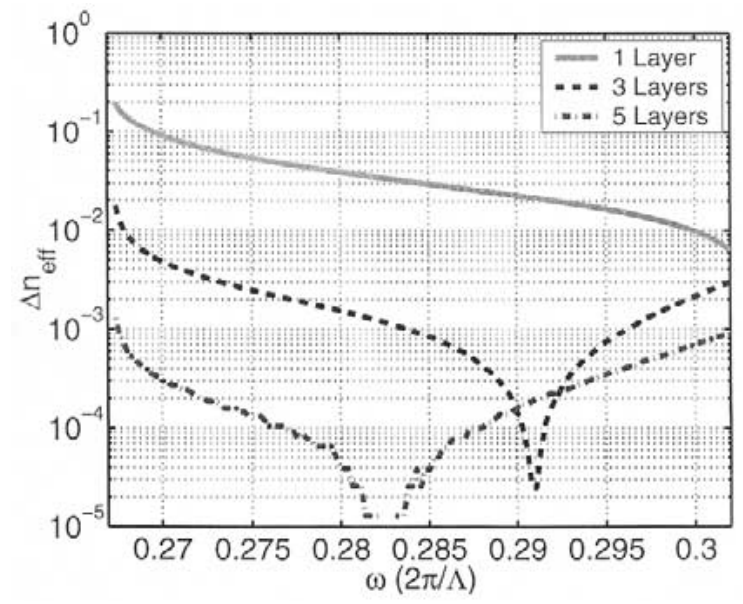

Fig. 7. The deviation of the effective indexes obtained using various inner core region layers. We use $n_{\text {eff }}^{N}$ to denote the effective index obtained using a $N$ layer core region. The curves labeled "1 layer," "3 layers," and "5 layers" represent, respectively, $\left|n_{\text {eff }}^{1}-n_{\text {eff }}^{7}\right|,\left|n_{\text {eff }}^{3}-n_{\text {eff }}^{7}\right|$, and $\left|n_{\text {eff }}^{5}-n_{\text {eff }}^{7}\right|$. The parameters of the Bragg fiber are given in the caption of Fig. 5 .

At the end of Section II-C, we described how to find the field distribution using the asymptotic approach. Basically, we must first obtain the propagation constant using (39). Substituting the result into (40) and (41), we obtain the modal amplitude coefficients in the first layer of the cladding region (i.e., $f_{\mathrm{TM}}$ and $f_{\mathrm{TE}}$ ) and those in the center air core (i.e., $\mathcal{A}_{\mathrm{TM}}$ and $\mathcal{C}_{\mathrm{TE}}$ ), respectively. Then, the cladding fields are easily found from (10) and (11), and the core fields are obtained by applying (6) and (7) repeatedly. We apply this algorithm to study the field distribution of the guided Bragg fiber mode at $\omega=0.286(2 \pi c / \Lambda)$. Using a core region of five layers, we find the propagation constant to be $\beta=0.128(2 \pi / \Lambda)$. The field distribution given by this asymptotic approach is represented by the solid lines in Fig. 8.

Having obtained the field distribution using the asymptotic algorithm, naturally, we would like to know how accurate the asymptotic approximation works without having to check asymptotic results using either the orginal algorithm by Yeh et al. or the FDTD method. We notice that the essence of the whole asymptotic algorithm is that the field distribution in the cladding region can be well described in the asymptotic limit. As long as this condition is satisfied, the asymptotic approach should provide a satisfactory description of the guided mode. To check the accuracy of the asymptotic approximation in the cladding region, we can calculate the exact field distribution in the cladding region by repeatedly applying (6) and (7). We use the phrase exact solution to denote results obtained this way. In other word, to find the exact solution, we still need to find $\mathcal{A}_{\mathrm{TM}}$ and $\mathcal{C}_{\mathrm{TE}}$ using (39), (40), and (41), as described previously. The only difference between the exact solution and the asymptotic solution is that, for the exact solution, the field distribution in the entire Bragg fiber is obtained from (6) and (7). Consequently, within the core region, the exact solution and the asymptotic solution are the same. In the cladding region, the two solutions differ from each other and their difference indicates how well the asymptotic approximation works. In Fig. 8, the exact solutions are represented by the dots. As expected, the exact solution and the asymptotic solution are the same within the core region. However, even in the cladding region, the difference between the
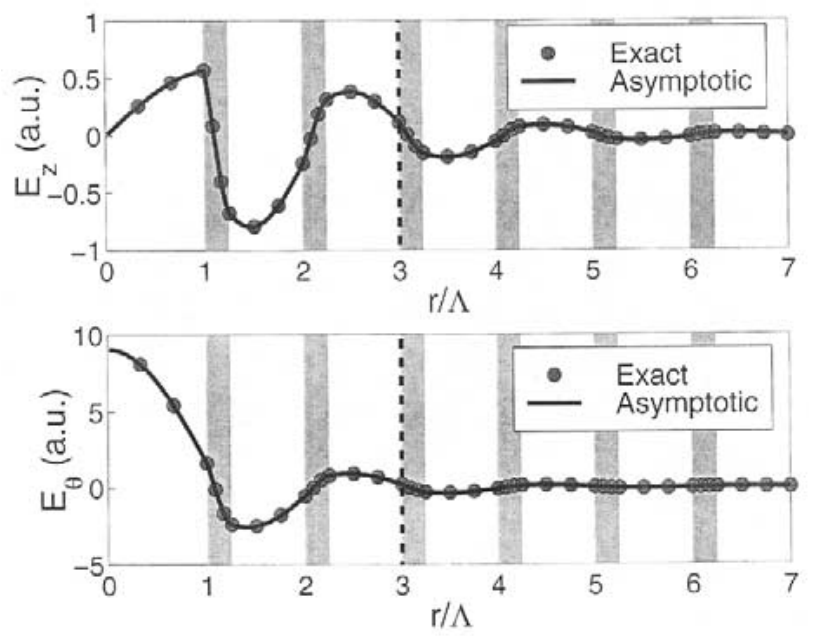

(a)
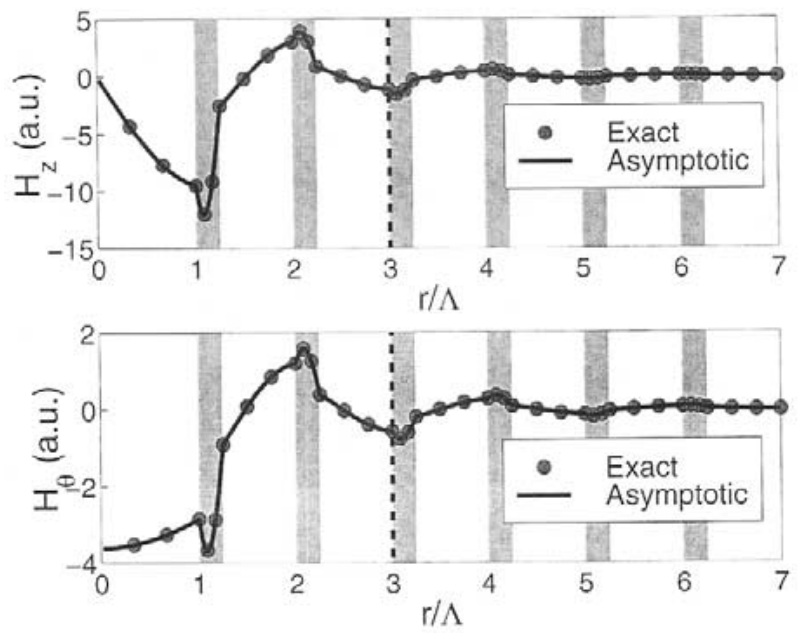

(b)

Fig. 8. The electromagnetic field distribution of the guided Brag fiber mode with (a) $E_{z}$ and $E_{\theta}$ components and (b) $H_{z}$ and $H_{\theta}$ components. The frequency of the mode is $\omega=0.286(2 \pi c / \Lambda)$. Using a five-layer core region, we find the effective modal index to be $n_{\text {eff }}=0.447$. The interface between the core region and cladding region is indicated by dash line. The exact solutions are obtained using (6) and (7) only. The asymptotic solutions are obtained using (6) and (7) in the core region and (10) and (11) in the cladding region.

two solutions are very small. Thus, we can conclude that the asymptotic algorithm gives an accurate description of the field distribution of the guided mode.

As a final remark, we notice that the free-space wavelength of the mode is $\lambda=3.5 \Lambda$. The ratio of the air core radius and the photon wavelength is only 0.286 . Inspecting Figs. 7 and 8, we find it quite amazing that for such small air core radius, the asymptotic approximation with five inner core layers works well.

\section{CONCLUSION}

In this paper, we proposed an asymptotic matrix theory to calculate modal dispersion, field distribution and radiation loss of any cylindrically symmetric dielectric geometries surrounded by Bragg cladding layers. This formalism is applied to analyze an air core Bragg fiber. The results are compared with those obtained from a 2-D FDTD algorithm, and good agreement between the two approaches is found. 


\section{REFERENCES}

[1] P. Yeh, A. Yariv, and E. Marom, "Theory of Bragg fiber," J. Opt. Soc. Amer., vol. 68, pp. 1196-1201, 1978.

[2] Y. Fink, D. J. Ripin, S. Fan, C. Chen, J. D. Joannopoulos, and E. L. Thomas, "Guiding optical light in air using an all-dielectric structure," J. Lightwave Technol., vol. 17, pp. 2039-2041, Nov. 1999.

[3] M. Miyagi, A. Hongo, Y. Aizawa, and S. Kawakami, "Fabrication of germanium-coated nickel hollow waveguides for infrared transmission," Appl. Phys. Lett., vol. 43, pp. 430-432, 1983.

[4] N. Croitoru, J. Dror, and I. Gannot, "Characterization of hollow fibers for the transmission of infrared radiation," Appl. Opt., vol. 29, pp. $1805-1809,1990$.

[5] R. F. Cregan, B. J. Mangan, J. C. Knight, T. A. Birks, P. S. J. Russell, P. J. Roberts, and D. C. Allan, "Single-mode photonic band gap guidance of light in air," Science, vol. 285, pp. 1537-1539, 1999.

[6] M. Ibanescu, Y. Fink, S. Fan, E. L. Thomas, and J. D. Joannopoulos, "An all-dielectric coaxial waveguide," Science, vol. 289, pp. 415-419, 2000.

[7] A. Yariv, Optical Electronics in Modern Communications. Oxford, U.K.: Oxford Univ. Press, 1997.

[8] P. Yeh and A. Yariv, "Bragg reflection waveguides," Opt. Commun., vol. 19, pp. 427-430, 1976

[9] Y. Xu, R. K. Lee, and A. Yariv, "Asymptotic analysis of Bragg fibers," Opt. Lett., vol. 25, pp. 1756-1758, 2000.

[10] J. Mathews and R. L. Walker, Mathematical Methods of Physics. Reading, MA: Addison-Wesley, 1970

[11] K. S. Yee, "Numerical solution of initial boundary value problems involving Maxwell's equations in isotropic media," IEEE Trans. Antennas Propagat., vol. AP-14, pp. 302-307, 1966.

[12] M. Plihal and A. A. Maradudin, "Photonic band structure of two-dimensional systems: The triangular lattice," Phys. Rev. B, vol. 44, pp. 8565-8571, 1991.
[13] F. Zepparelli, P. Mezzanotte, F. Alimenti, L. Roselli, R. Sorrentino, G. Tartarini, and P. Bassi, "Rigorous analysis of 3D optical and optoelectronic devices by the compact-2D-FDTD method," Opt. Quantum Electron., vol. 31, pp. 827-841, 1999.

[14] N. J. Doran and K. J. Blow, "Cylindrical Bragg fibers: A design and feasibility study for optical communications," J. Lightwave Technol., vol. LT-1, pp. 588-590, 1983.

[15] J. P. Berenger, "A perfectly matched layer for the absorption of electromagnetic waves," J. Computat. Phys., vol. 114, pp. 185-200, 1994.

[16] S. D. Gedney, "An anisotropic perfectly matched layer-absorbing medium for the truncation of FDTD lattices," IEEE Trans. Antennas Propagat., vol. 44, pp. 1630-1639, Dec. 1996.

Yong $\mathrm{Xu}$, photograph and biography not available at the time of publication.

George X. Ouyang, photograph and biography not available at the time of publication.

Reginald K. Lee (S'97-M'00), photograph and biography not available at the time of publication.

Amnon Yariv (S'56-M'59-F'70-LF'95), photograph and biography not available at the time of publication. 